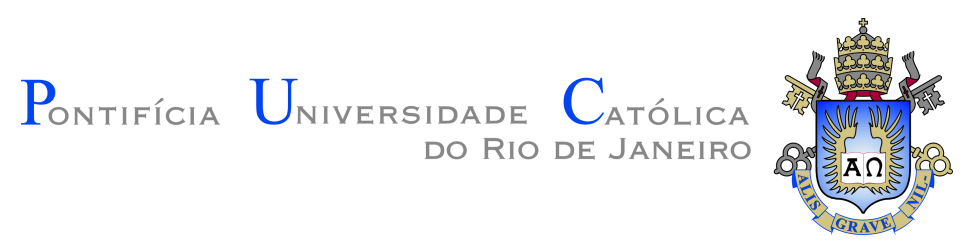

David Nuñez Cuadrado

Antenna Selection in MIMO-OFDM systems

Dissertação de Mestrado

Dissertation presented to the Programa de Pós-graduação em Engenharia Elétrica da PUC-Rio in partial fulfillment of the requirements for the degree of Mestre em Engenharia Elétrica.

Advisor : Prof. Raimundo Sampaio-Neto

Co-advisor: Dr. João Cal-Braz 


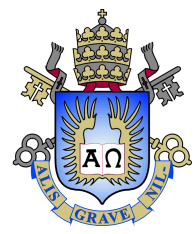

David Nuñez Cuadrado

\section{Antenna Selection in MIMO-OFDM systems}

Dissertation presented to the Programa de Pós-graduação em Engenharia Elétrica da PUC-Rio in partial fulfillment of the requirements for the degree of Mestre em Engenharia Elétrica. Approved by the Examination Committee.

Prof. Raimundo Sampaio-Neto

Advisor

Centro de Estudos em Telecomunicações PUC-Rio

Dr. João Cal-Braz

Co-advisor

Inmetro

Dr. Rodrigo Pereira David

Inmetro

Dr. Fabian David Backx

Instituto de Pesquisas de Marinha - IPqM

Rio de Janeiro, March the 23rd, 2020 
All rights reserved.

\section{David Nuñez Cuadrado}

Graduated in Telecommunications and Electronic Engineering from University "Marta Abreu" of Las Villas, Cuba, 2012.

Bibliographic data

Nuñez Cuadrado, David.

Antenna Selection in MIMO-OFDM systems / David Nuñez Cuadrado; advisor: Raimundo Sampaio-Neto; coadvisor: João Cal-Braz. - 2020.

67 f. : il. color. ; $30 \mathrm{~cm}$

Dissertação (mestrado) - Pontifícia Universidade Católica do Rio de Janeiro, Departamento de Engenharia Elétrica, 2020.

\section{Inclui bibliografia}

1. Engenharia Elétrica - Teses. 2. MIMO-OFDM;. 3. Seleção de antenas;. 4. Equalização linear;. 5. Pré-codificação linear;. 6. Desempenho;. I. Sampaio-Neto,R.. II. Cal-Braz, J.. III. Pontifícia Universidade Católica do Rio de Janeiro. Departamento de Engenharia Elétrica. IV. Título.

CDD: 621.3 


\section{Acknowledgments}

First of all, I wish to thank to my life coaches, my parents because I owe it all to you. Many Thanks!

I want to thank to my wife, because she has been extremely supportive of me throughout this entire process and has made countless sacrifices to help me get to this point.

A special thanks to my family. Words cannot express how grateful I am to all of you.

I would like to express my sincere gratitude to my advisor Prof. Raimundo Sampaio-Neto for the continuous support, for his patience, motivation, and immense knowledge. The door to his office was always open whenever I ran into a trouble spot or had a question about my research. I could not have imagined having a better advisor and mentor for me.

I am also grateful to my co-advisor Dr. João Cal-Braz for their support in overcoming numerous obstacles I have been facing through my research.

To all my friends, who directly or indirectly contributed to this work. Especially to the family of Cubans I met in Brazil, with whom I shared incredible moments.

I would also like to thank to PUC-Rio and CETUC for the support.

This study was financed in part by the Coordenação de Aperfeiçoamento de Pessoal de Nível Superior - Brasil (CAPES) - Finance Code 001 


\section{Abstract}

Nuñez Cuadrado, D.; Sampaio-Neto,R. (Advisor); Cal-Braz, J. (CoAdvisor). Antenna Selection in MIMO-OFDM systems. Rio de Janeiro, 2020. 67p. Dissertação de mestrado - Departamento de Engenharia Elétrica, Pontifícia Universidade Católica do Rio de Janeiro.

This thesis focuses on the combination of two well-known effective technologies used in many of today's communication networks, Orthogonal Frequency Division Multiplexing (OFDM) and Multiple-Input MultipleOutput (MIMO). It unites the well-known advantages of MIMO communication, such as high data rates and improved link reliability to the characteristics of OFDM, known as intersymbol-interference-free communication and simple frequency-domain equalization. This work presents in detail the signal modelling of MIMO-OFDM system and the adequate processing at the receiver to perform decoupled detections per OFDM subsymbol. In addition, antenna selection strategies are proposed to increase system performance by exploiting some scenarios when the transmitter or receiver is equipped with more antennas than the number of radiofrequency $(\mathrm{RF})$ chains. These procedures are based on the specific metric for the current scenario. The performance of the system is evaluated employing linear equalization or precoding techniques, such as Zero-Forcing (ZF) and Minimum Mean Square Error (MMSE). Simulation results evidence that antenna selection strategies result in significant system performance improvement.

\section{Keywords}

MIMO-OFDM; Antenna selection; Linear equalization; Linear precoding; Performance; 


\section{Resumo}

Nuñez Cuadrado, D.; Sampaio-Neto,R.; Cal-Braz, J.. Seleção de antenas em sistemas MIMO-OFDM. Rio de Janeiro, 2020. 67p. Dissertação de Mestrado - Departamento de Engenharia Elétrica, Pontifícia Universidade Católica do Rio de Janeiro.

Esta dissertação enfoca a combinação de duas conhecidas e eficazes tecnologias usadas em muitas redes de comunicação atuais, as técnicas de transmissão Orthogonal Frequency Division Multiplexing (OFDM) e Multiple-Input Multiple-Output (MIMO). Sistemas MIMO-OFDM unem as vantagens conhecidas da comunicação MIMO, como altas taxas de dados e ampliação da confiabilidade do link, com as características marcantes da transmissão OFDM, como comunicação livre de interferência entre símbolos e a equalização simples no domínio da frequência. Este trabalho apresenta em detalhes a modelagem de sinais do sistema MIMO-OFDM e o processamento adequado no receptor para realizar detecções desacopladas dos sub-símbolos OFDM. Além disso, estratégias de seleção de antenas são propostas para aumentar o desempenho do sistema, explorando cenários em que o transmissor ou receptor está equipado com mais antenas do que o número de cadeias de radiofrequência (RF). Esses procedimentos são baseados em métricas específicas propostas para cada cenário. A avaliação de desempenho inclui sistemas equipados com técnicas de equalização linear e sistemas com pré-codificadores, como Zero-Forcing (ZF) e Minimum Mean Square Error (MMSE). Os resultados de simulação evidenciam que as estratégias de seleção da antena resultam em uma melhoria significativa no desempenho do sistema.

\section{Palavras-chave}

MIMO-OFDM; Seleção de antenas; Equalização linear; Précodificação linear; Desempenho; 


\section{Table of contents}

1 Introduction $\quad 14$

2 Fundamentals of MIMO-OFDM systems $\quad 17$

2.1 System Model 17

$\begin{array}{ll}2.1 .1 & \text { Single-user MIMO-OFDM }\end{array}$

$\begin{array}{ll}2.1 .1 .1 & 19 \\ 2.1 .2 & \text { Non-precoded case }\end{array}$

2.1.1.2 Precoded case 21

2.1.2 Multi-User MIMO-OFDM 22

2.2 Mobile radio channel characterization 23

2.2.1 Large-scale fading 24

2.2.2 Small-scale fading 25

$\begin{array}{lll}2.3 & \text { Detection Techniques } & 27\end{array}$

2.3.1 Zero-Forcing Detection, single-user 27

2.3.2 Minimum Mean Square Error Detector, single-user 28

2.4 Precoding for multi-user system 28

$\begin{array}{lll}2.4 .1 & \text { Zero-Forcing } & 29\end{array}$

2.4.2 Minimum Mean Square Error 30

3 Transmit Antennas Selection $\quad 32$

3.1 SU-MIMO-OFDM system (non-precoded) 33

3.1.1 Transmit Antenna Selection Model for SU-MIMO-OFDM systems 33

3.1.2 Mutual Information Approach (MIA) 33

3.1.3 Mean Square Error (MSE) Minimizing Approach 34

3.1.3.1 MSE Minimizing approach in ZF-equalized receiver 34

3.1.3.2 MSE Minimizing approach in MMSE-equalized receiver $\quad 35$

$\begin{array}{lll}3.1 .4 & \text { Simulation Results } & 35\end{array}$

3.1.4.1 ZF and MMSE for MIA 36

3.1.4.2 ZF and MMSE for MSE approach 38

3.1.4.3 Comparisons 39

3.2 MU-MIMO-OFDM system (precoded) 41

3.2.1 Transmit Antenna Selection Model for MU-MIMO-OFDM systems 41

3.2.2 Transmit Antenna Selection Approach for ZF Precoding 41

3.2.3 Transmit Antenna Selection Approach for MMSE Precoding 42

3.2.4 Simulation Results 43

3.2.4.1 ZF Precoding 43

3.2.4.2 MMSE Precoding 46

4 Receive Antennas Selection $\quad 47$

4.1 SU-MIMO-OFDM system (non-precoded) 48

4.1.1 Receive Antenna Selection Model for SU-MIMO-OFDM systems 48

4.1.2 Receive Antenna Selection using MIA 48

4.1.3 Receive Antenna Selection minimizing the MSE 49

4.1.3.1 MSE Minimizing in ZF-equalized receiver 49

4.1.3.2 MSE Minimizing in MMSE-equalized receiver 49 
4.1.4 Simulation Results $\quad 50$

4.1.4.1 ZF and MMSE for MIA 50

4.1.4.2 ZF and MMSE for MSE approach $\quad 52$

4.1.4.3 Comparisons 53

4.2 MU-MIMO-OFDM system (precoded) 55

4.2.1 Receive Antenna Selection Model for MU-MIMO-OFDM systems 55

4.2.2 Receive Antenna Selection Approach for ZF Precoding 55

4.2.3 Receive Antenna Selection Approach for MMSE Precoding 56

4.2.4 Simulation Results $\quad 57$

4.2.4.1 ZF Precoding $\quad 57$

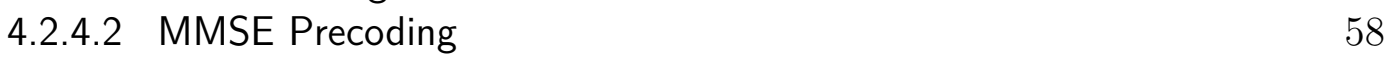

5 Conclusions and Future Work $\quad 60$

Bibliography $\quad 62$

$\begin{array}{lll}\text { A Energy Relations for Multi-User } & 66\end{array}$

$\begin{array}{lll}\text { B Energy Relations for Single-User } & 67\end{array}$ 


\section{List of figures}

$\begin{array}{lll}\text { Figure 2.1 } & \text { Block diagram of OFDM systems } & 18\end{array}$

Figure 2.2 Block diagram of the baseband discrete model of a SU-

MIMO-OFDM system.

Figure 2.3 Data-matrix $\mathcal{D}$ for MIMO-OFDM system.

19

Figure 2.4 The $\mathbf{r}_{m}$ vectors are rearranged from vector $\mathbf{y}$.

Figure 2.5 Data-matrix $\mathcal{D}$ for SU-MIMO-OFDM system (nonprecoded case).

Figure 2.6 Block diagram of the baseband discrete model of a MUMIMO-OFDM system.

Figure 3.1 MIMO-OFDM system with antenna selection at the transmitter.

Figure 3.2 BER vs. SNR [dB] for transmit antenna selection using Mutual Information Approach, ZF equalizer, and considering a different number of available antennas at the transmitter.

Figure 3.3 BER vs. SNR $[\mathrm{dB}]$ for transmit antenna selection using Mutual Information Approach, MMSE equalizer, and considering a different number of available antennas at the transmitter.

Figure 3.4 BER vs. SNR [dB] for transmit antenna selection using MSE Approach, ZF equalizer, and considering a different number of available antennas at the transmitter.

Figure 3.5 BER vs. SNR $[\mathrm{dB}]$ for transmit antenna selection using MSE Approach, MMSE equalizer, and considering a different number of available antennas at the transmitter.

Figure 3.6 BER vs. SNR [dB] for transmit antenna selection using the proposed methods (MIA and MSE) and random selection. Results were obtained employing ZF equalizer.

Figure 3.7 BER vs. SNR [dB] for transmit antenna selection using the proposed methods (MIA and MSE) and random selection. Results were obtained employing MMSE equalizer.

Figure 3.8 BER vs. SNR $[\mathrm{dB}]$ for $N_{T}=10, N_{R F}=10, N_{R}=3$, $K=2$ and $L=2$ in ZF-precoded system.

Figure 3.9 BER vs. SNR $[\mathrm{dB}]$ for transmit antenna selection using $\mathrm{ZF}$ precoding, $K=2$, and considering a different number of antennas and RF chains available at the transmitter.

Figure 3.10 BER vs. SNR [dB] for transmit antenna selection using MMSE precoding, $K=2$, and considering a different number of antennas and RF chains available at the transmitter.

Figure 4.1 MIMO-OFDM system with antenna selection at the receiver.

Figure 4.2 BER vs. SNR [dB] for receive antenna selection using Mutual Information Approach, ZF equalizer, and considering a different number of available antennas at the receiver. 
Figure 4.3 BER vs. SNR $[\mathrm{dB}]$ for receive antenna selection using Mutual Information Approach, MMSE equalizer, and considering a different number of available antennas at the receiver.

Figure 4.4 BER vs. SNR [dB] for receive antenna selection using

MSE Approach, ZF equalizer and considering different number of available antennas at the receiver.

Figure 4.5 BER vs. SNR [dB] for receive antenna selection using MSE Approach, MMSE equalizer and considering different number of available antennas at the receiver.

Figure 4.6 BER vs. SNR [dB] for receive antenna selection using the proposed methods (MIA and MSE) and random selection. Results were obtained employing ZF equalizer.

Figure 4.7 BER vs. SNR [dB] for receive antenna selection using the proposed methods (MIA and MSE) and random selection. Results were obtained employing MMSE equalizer.

Figure 4.8 BER vs. SNR [dB] for receive antenna selection using $\mathrm{ZF}$ precoding, $K=3$, and considering a different number of available antennas at the receiver.

Figure 4.9 BER vs. SNR [dB] for receive antenna selection using MMSE precoding, $K=3$, and considering a different number of available antennas at the receiver. 


\section{List of Abreviations}

3D - Three-Dimensional

3GPP - Third Generation Partnership Project

$4 \mathrm{G}$ - Fourth-Generation

$5 \mathrm{G}$ - Fifth-Generation

AR - Augmented Reality

BER - Bit-Error Rate

BS - Base Station

CP - Cyclic-Prefix

CSI - Channel State Information

DFT - Discrete Fourier Transform

FDD - Frequency Division Duplexing

FFT - Fast Fourier Transform

IEEE - Institute of Electrical and Electronics Engineers

LOS - Line of Sight

MIA - Mutual Information Approach

MIMO - Multiple-Input Multiple-Output

MMSE - Minimum Mean Square Error

MSE - Mean Square Error

MU - Multi-user

MUI - Multi-user Interference

OFDM - Orthogonal Frequency Division Multiplexing

PSK - Phase Shift Keying 
QAM - Quadrature Amplitude Modulation

QoE - Quality of Experience

QPSK - Quadrature Phase Shift Keying

RAN - Random Selection

RF - Radio Frequency

SINR - Signal-to-Interference-plus-Noise Ratio

SNR - Signal-to-Noise Ratio

STBC - Space-Time Block Codes

SU - Single-User

TDD - Time Division Duplexing

UE - User Equipment

UHD - Ultra High Definition

VR - Virtual Reality

Wi-Fi - Wireless Fidelity

ZF - Zero Forcing 
"By recording your dreams and goals on paper, you set in motion the process of becoming the person you most want to be. Put your future in good hands - your own."

Mark Victor Hansen 


\section{1 \\ Introduction}

Wireless communication systems have experienced an accelerated evolution in the last decades caused by the stringent requirements in terms of data rates, latency and energy efficiency. Each generation of mobile technology has also provided significant performance enhancements.

The fast penetration of the fourth-generation $(4 \mathrm{G})$ mobile network has allowed new mobile services and applications to be launched. It is expected to promote many others, such as Augmented Reality (AR), Virtual Reality (VR), Ultra High Definition (UHD) video (4K/8K), and glass-free 3D display [1]. These applications demand higher capacities and efficiency from mobile communication networks. Industrial Internet and automotive demands also require much lower transmission latencies and higher reliability. Ultra high throughput, ubiquitous connectivity, energy efficiency, low-cost devices and quality of experience (QoE) are just some of the requirements that the next generation wireless needs to achieve [2].

On the other hand, as a wide-area wireless technology, the fifthgeneration (5G) mobile network is growing, and it should provide an improvement over the current 4G in capacity (between 100 and 1000 times as predicted) and other functionalities so that it continues to satisfy the growing user expectations of QoE. It also promises improvements over $4 \mathrm{G}$ in terms of denser network coverage, faster download time, HD-video streaming and so on. The endless enormous growth of data traffic volume is one of the main drivers behind $5 \mathrm{G}$, and the annual $25-50 \%$ growth of data rate is expected to continue until 2030 and beyond [3]. The challenge is to find and develop the wireless communication network and technologies that will support massive data traffic volume in the coming years.

Among the recent developments achieved so far, Orthogonal Frequency Division Multiplexing and Multiple-Input Multiple-Output figure in most of the current communication technologies, due to their indisputable effectiveness.

International mobile telephony standards organization has settled OFDM as the main waveform for the $5 \mathrm{G}$ mobile service radio access [4], endorsing the well-known advantages of OFDM communication. OFDM technique is a 
spectrally efficient modulation scheme that transforms a broadband channel with frequency-selective fading into a set of parallel narrowband channels with frequency-flat fading, avoiding the occurrence of intersymbol interference and simplifying the system in terms of equalization [5].

MIMO communication is an emerging technique that offers various advantages through the deployment of multiple antennas at the communicating nodes. Channel capacity that increases linearly with system size is achieved, resulting in high data rates by the exploitation of the spatial domain. Secondly, the diversity gain experienced by the multiantenna systems offers improved reliability of the transmission link, mitigating the deleterious effect of fading [6]. The use of a large number of antennas at the base station (BS) to achieve more diversity is also known as massive MIMO. These systems are employed to serve a high number of users at the same time without requiring extra bandwidth resources, which has been extensively studied in the last decade $[7,8]$.

Most MIMO literature assumes frequency-flat fading channel and, when stated otherwise, assumes the use of OFDM transmission to build an equivalent system model that reverts the frequency-selective environment back into frequency-flat, taking the particularities of MIMO-OFDM system for granted or leaving them unspecified $[9,10]$.

Thus, the combination of both techniques results in a system with enhanced performance and more resilient to communication errors. The main drawback of a MIMO-OFDM implementation is the cost, in terms of size, power consumption and hardware complexity, which is scaled with the number of antennas since there is a radio frequency chain and an OFDM modulator associated to each antenna element [11]. In order to overcome this challenge, strategies that can lower the energy consumption and the cost of implementation and operation are required.

Antenna selection strategies have been considered in several frequencyflat MIMO scenarios as viable solutions that reduce the hardware complexity through the use of a number of RF chains smaller than the number of available antennas in the system [11-13]. The underlying idea is to use a reduced number of RF chains and, based on the current channel characteristics, choose a subset of available antennas more adequate to communication to which the RF chains should be connected.

Several antenna selection schemes have been developed, mainly for singleuser MIMO communications. The selection criteria range from minimizing the symbol error rate [12] or the channel matrix condition number, to maximizing channel capacity [14], among others. When the multi-user scenario is consid- 
ered, relevant works consider the maximization of the signal-to-leakage and noise ratio $[15],[16]$.

This dissertation aims to propose an antenna selection strategy for singleuser (SU) and multi-user (MU) MIMO-OFDM systems. The proposed antenna selection approaches are formulated for the transmit or receive antenna selection. The scenarios analyzed here were studied in depth in order to propose various mechanisms to improve the antenna selection techniques existing in the literature. Typical scenarios in communication systems commonly handle equalization and precoding techniques employed in this dissertation. Between the main contributions of this work, we can highlight the development of optimal antenna selection strategies, aiming at the minimization of the detection error probability of the system.

The chapters of this thesis are organized as follows: in Chapter 2, the basic concepts for MIMO-OFDM systems are studied, starting with the signal model using a different approach to the one used in the literature. Then, a review of the mobile radio channel characterization is shown. Finally, the main detection and precoding techniques are presented.

In Chapter 3 a transmit antenna selection strategy is developed for singleuser and multi-user MIMO-OFDM systems. The mathematical representation that describes the system and expressions for a given metric are presented. Simulation results to evaluate the performance of the proposed selection schemes, with Zero-Forcing (ZF) and Minimum Mean Square Error (MMSE) techniques, are also presented.

In Chapter 4, the problem of receive antenna selection is addressed, describing the mathematical model of the system. Simulation results to evaluate the performance of the proposed strategies are provided, when ZF and MMSE techniques are employed.

Conclusions of this work are presented and future path for this research topic are discussed in Chapter 5.

Throughout this work, we have employed the following notation: boldface letters will be used for matrices (capital letters) and vectors (lowercase letters); $(\cdot)^{*},(\cdot)^{T}$ and $(\cdot)^{H}$ denote conjugate, transpose and Hermitian (conjugate transpose), respectively; $[A]^{\dagger}$ is the pseudo-inverse of matrix $\mathrm{A} ; \mathbb{E}[\cdot]$ is used to represent the expected value of a scalar or vectorial random variable and $\operatorname{Tr}\{\cdot\}$ to represent the trace of a matrix. $\mathbf{I}_{N}$ denotes the $N \times N$ identity matrix. Further, $\operatorname{diag}\{\mathbf{v}\}$ stands for a diagonal matrix with the components of vector $\mathbf{v}$ on its main diagonal. We have used i.i.d to refer to independent, identically distributed random quantities. 


\section{2}

\section{Fundamentals of MIMO-OFDM systems}

In this Chapter, a general overview of MIMO-OFDM systems is shown. A more comprehensive model, compared to the one found in the current literature, is presented. A brief description of the most important channel effects is also outlined, as well as the equalizers and precoders used in this work.

\section{1}

\section{System Model}

OFDM transmission scheme has been applied in a variety of wireless communication systems in the last decades due to the advantages over conventional single-carrier modulation schemes when enduring frequency-selective fading channels.

By splitting the information to be transmitted into blocks, intersymbol interference is replaced by intercarrier and interblock interferences, which are dealt by the OFDM scheme.

Under the consideration that the frequency-selective channel is timeinvariant within the transmission block, OFDM system is exempt from intercarrier interference induced by multipath propagation. Also, the interblock interference is perfectly removed if a guard interval with sufficient length is appended to the transmitted block.

The simplicity of equalization is also an important feature of OFDM [5]. If the cyclic-prefix (CP) guard interval is used, the Fourier bases that compose the direct and inverse Discrete Fourier Transform (DFT) matrices used at the transmitter and receiver sides by OFDM form the eigenbases of the block circulant channel matrix available at the receiver after CP removal. As a result, the equalizer is implemented by a single-tap filter.

Within this work, CP guard interval is considered, rather than the zeropadded guard interval.

Let $\mathbf{d}$ be the data vector with length $M$ (number of sub-symbols in the OFDM block, belonging to a complex signal constellation, e.g. PSK, QAM, with symbols drawn from the set $\mathcal{M}$ ), to be transmitted through an $L$-tap 
frequency selective dispersive channel and received in the presence of additive noise. Figure 2.1 shows the block diagram of an OFDM system.

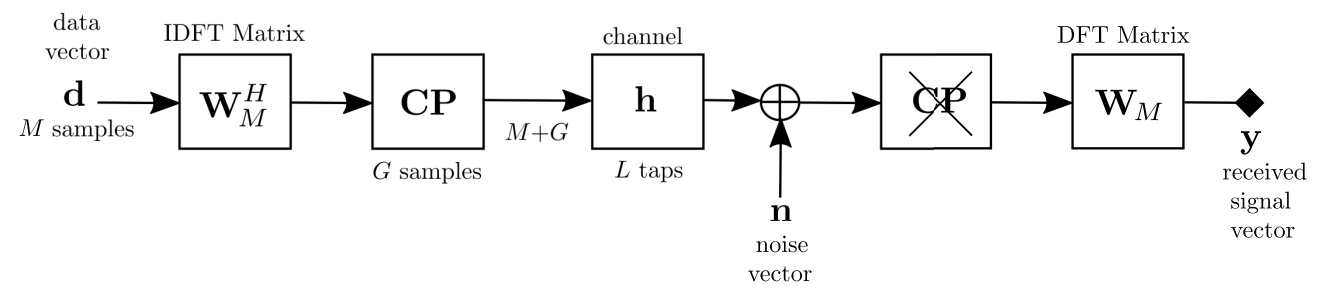

Figure 2.1: Block diagram of OFDM systems

It is well known that, if the length of the cyclic prefix $G$ is not less than $L-1$, and the channel remains fixed for at least a period of OFDM block, the received vector (after cyclic prefix removal and the Fast Fourier Transform operation) can be expressed in the form:

$$
\mathbf{y}=\mathbf{D d}+\mathbf{n},
$$

where $\mathbf{D}$ is a diagonal matrix that contains in its main diagonal the components of the discrete channel frequency response,

$$
\mathbf{D}=\left[\begin{array}{cccc}
\mathrm{D}_{1} & 0 & \ldots & 0 \\
0 & \mathrm{D}_{2} & \ldots & 0 \\
\vdots & \vdots & \ddots & 0 \\
0 & 0 & \ldots & \mathrm{D}_{M}
\end{array}\right]=\operatorname{diag}\left(\sqrt{M} \mathbf{W}_{M} \mathbf{h}_{e}\right)
$$

In turn, $\mathbf{W}_{M}$ is the normalized DFT matrix $\left(\mathbf{W}_{M}^{H} \mathbf{W}_{M}=\mathbf{I}_{M}\right)$ and vector $\mathbf{h}_{e}$ contains the baseband discrete channel impulse response $\mathbf{h}=\left[h_{1}, h_{2}, \ldots, h_{L}\right]^{\mathrm{T}}$ padded with $M-L$ zeros [5].

\subsection{1}

\section{Single-user MIMO-OFDM}

MIMO systems take advantage of the spatial diversity that is obtained by spatially separated antennas in a rich multipath scattering environment. Codes applied to the transmitted data can be used to increase the spatial diversity, such as space-time block codes (STBC). Alternatively, the transmission of independent streams in parallel by the antennas yields a linear increase in capacity. This is called spatial multiplexing and is the focus of the strategies developed herein. MIMO-OFDM combines the advantages of both strategies, justifying its use as the dominant air interface of most modern broadband communication technologies, such as wireless local area networks (WiFi IEEE 802.11ax) [17] and cellular communication (5G 3GPP Release-15) [18]. 


\subsubsection{1}

\section{Non-precoded case}

The system under consideration (SU-MIMO-OFDM) is represented in Figure 2.2. Both transmitter and receiver have multiple antennas. The discrete channel impulse response vectors $\mathbf{h}_{i j}, i=1,2, \ldots, N_{R}, j=1,2, \ldots, N_{T}$ are assumed i.i.d.

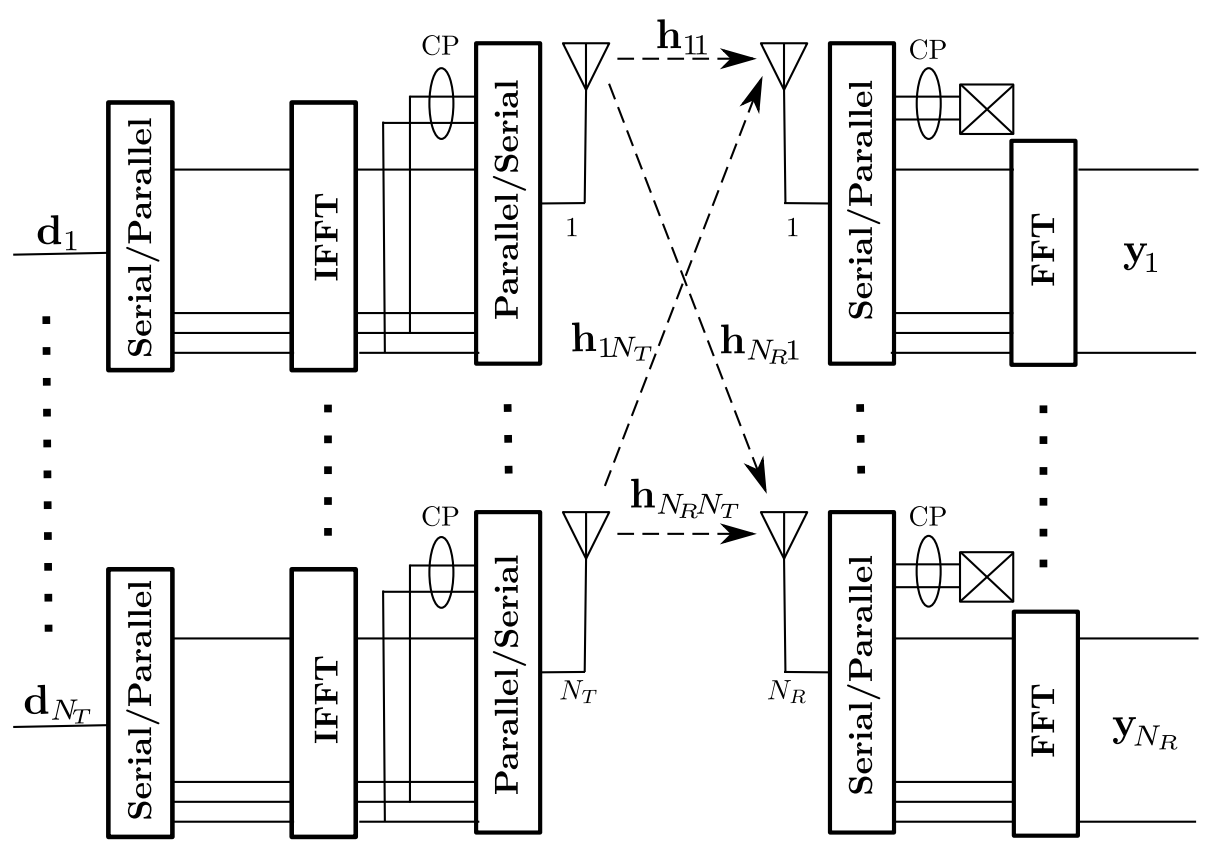

Figure 2.2: Block diagram of the baseband discrete model of a SU-MIMO-OFDM system.

The set of data vectors $\left\{\mathbf{d}_{1}, \mathbf{d}_{2}, \ldots, \mathbf{d}_{N_{T}}\right\}$ are arranged in rows forming the data-matrix $\mathcal{D}$, illustrated in Figure 2.3. The $N_{T}$ transmitted OFDM blocks are the columns of the matrix $\mathcal{T}=\mathbf{W}_{M}^{H} \mathcal{D}^{T}$. That means that at the $m$-th transmission, the vector transmitted by the $N_{T}$ transmitting antennas corresponds to $m$-th column of the transmission matrix $T=\mathcal{T}^{T}=\mathcal{D} \mathbf{W}_{M}^{*}$.

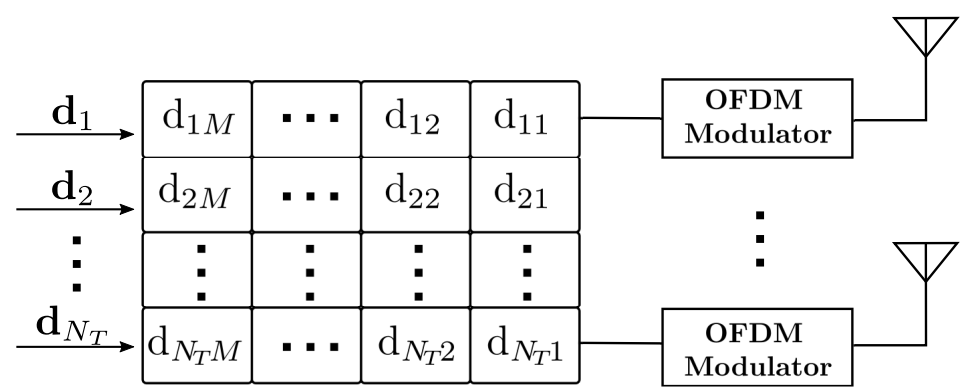

Figure 2.3: Data-matrix $\mathcal{D}$ for MIMO-OFDM system.

Considering (2-1), (2-2) and the model in Figure 2.2 we can express the signal at all receive antennas by the vector $\mathbf{y}=\left[\begin{array}{llll}\mathbf{y}_{1}^{T}, & \mathbf{y}_{2}^{T}, & \ldots & , \mathbf{y}_{N_{R}}^{T}\end{array}\right]^{T}$, $\mathbf{y}_{k} \in \mathbb{C}^{M \times 1}, k=1,2, \ldots, N_{R}, \quad$ as 


$$
\mathbf{y}=\mathbf{H d}+\mathbf{n}
$$

where $\mathbf{d}=\left[\begin{array}{llll}\mathbf{d}_{1}^{T}, & \mathbf{d}_{2}^{T}, \quad \ldots, \mathbf{d}_{N_{T}}^{T}\end{array}\right]^{T}, \mathbf{d}_{k} \in \mathcal{M}^{M \times 1}, k=1,2, \ldots, N_{T}$, and $\mathbb{E}\left[\mathbf{d}_{k} \mathbf{d}_{k}^{H}\right]=\mathrm{E}_{s} \mathbf{I}_{M}$, thus $\mathrm{E}_{s}$ corresponds to the average symbol energy. The $M N_{R} \times M N_{T}$ matrix $\mathbf{H}$ is comprised of the $N_{R} N_{T}$ diagonal matrices corresponding to the $N_{R} N_{T}$ channel vectors $\left\{\mathbf{h}_{i j}\right\}$. The noise vector $\mathbf{n}=$ $\left[\begin{array}{llll}\mathbf{n}_{1}^{T}, & \mathbf{n}_{2}^{T}, & \ldots & , \mathbf{n}_{N_{R}}^{T}\end{array}\right]^{T}$, where $\mathbf{n}_{l}=\left[\begin{array}{ll}\mathrm{n}_{l 1} & \mathrm{n}_{l 2} \ldots \mathrm{n}_{l M}\end{array}\right]^{T}, l=1,2, \ldots, N_{R}$, are complex Gaussian vectors, statistically independent with zero-mean and covariance matrix $\mathbf{K}_{\mathbf{n}}=\mathbb{E}\left[\mathbf{n}_{l} \mathbf{n}_{l}^{H}\right]=\sigma_{n}^{2} \mathbf{I}_{M}$. Expanding the above, we can express the system as

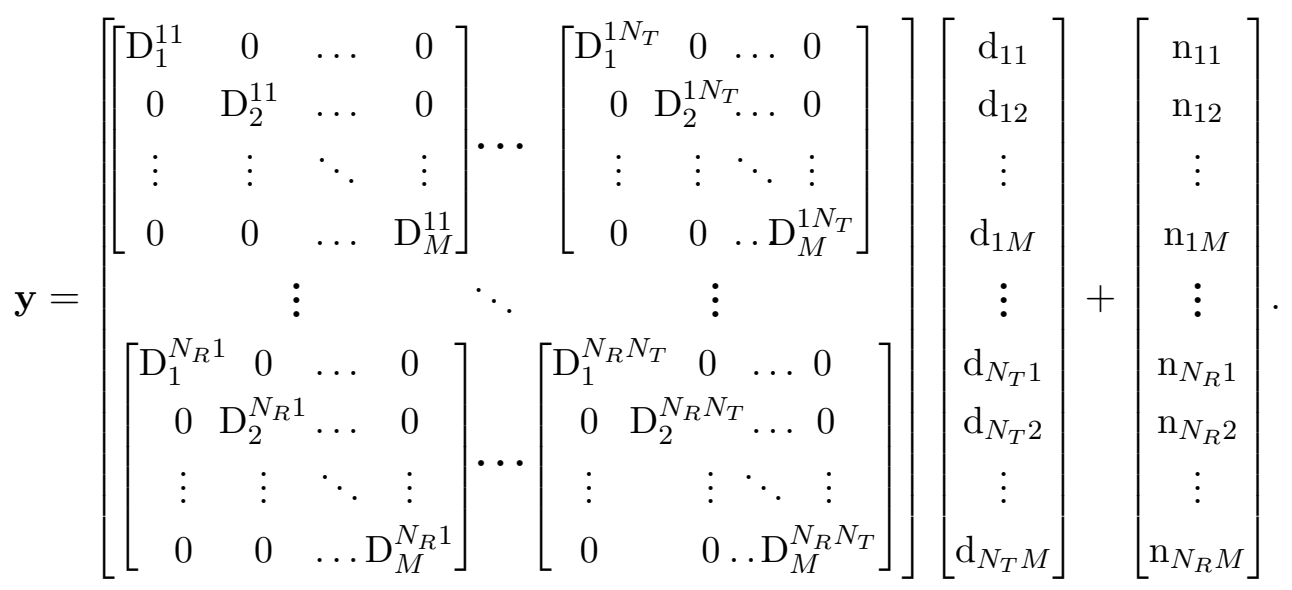

Considering the received signal $\mathbf{y}$ given by (2-3), classical suboptimum detection schemes (e.g. ZF and MMSE linear detectors) can be used to detect the data vector $\mathbf{d}$. However these schemes involve the $M N_{R} \times M N_{T}$ matrix $\mathbf{H}$ in (2-3) and may require the inversion of very large dimension matrices when the number of transmitting and receiving antennas or the length of the OFDM blocks is high.

Taking a closer look at expression (2-3) and matrix $\mathbf{H}$ in (2-4), we conclude that the components of the received vector $\mathbf{y}$ can be rearranged to yield $\tilde{\mathbf{y}}=\left[\begin{array}{llll}\mathbf{r}_{1} & \mathbf{r}_{2} & \ldots & \mathbf{r}_{M}\end{array}\right]^{T}$, where the vectors $\mathbf{r}_{m} \in \mathbb{C}^{N_{R} \times 1}, m=1,2, \ldots, M$, are the columns of the matrix shown in Figure 2.4.

Thus, the $\mathbf{r}_{m}$ vectors can be obtained as:

$$
\mathbf{r}_{m}=\mathbb{D}_{m} \mathbf{x}_{m}+\tilde{\mathbf{n}}_{m} \quad ; \quad m=1,2, . ., M
$$

where $\mathbf{x}_{m}=\left[\begin{array}{lll}\mathrm{d}_{1 m} & \mathrm{~d}_{2 m} \ldots \mathrm{d}_{N_{T} m}\end{array}\right]^{\mathrm{T}}$ is the $m$-th column of the data matrix $\mathcal{D}$ in Figure $2.5, \mathbb{D}_{m}$ is a $N_{R} \times N_{T}$ submatrix of $\mathbf{H}$ given by 


$$
\left.\mathbf{y}=\left[\begin{array}{c}
\mathbf{y}_{1} \\
\mathbf{y}_{2} \\
\vdots \\
\mathbf{y}_{N_{R}}
\end{array}\right]=\left[\begin{array}{cccc}
{\left[\begin{array}{cccc}
y_{11} & y_{12} & \ldots & y_{1 M}
\end{array}\right]^{T}} \\
{\left[\begin{array}{cccc}
y_{21} & y_{22} & \ldots & y_{2 M}
\end{array}\right]^{T}} \\
\vdots & \vdots & \vdots & \vdots \\
{\left[y_{N_{R} 1}\right.} & y_{N_{R} 2} & \ldots & y_{N_{R} M}
\end{array}\right]^{T}\right]
$$

Figure 2.4: The $\mathbf{r}_{m}$ vectors are rearranged from vector $\mathbf{y}$.

$$
\mathbb{D}_{m}=\left[\begin{array}{cccc}
\mathrm{D}_{m}^{11} & \mathrm{D}_{m}^{12} & \ldots & \mathrm{D}_{m}^{1 N_{T}} \\
\mathrm{D}_{m}^{21} & \mathrm{D}_{m}^{22} & \ldots & \mathrm{D}_{m}^{2 N_{T}} \\
\vdots & \vdots & \ddots & \vdots \\
\mathrm{D}_{m}^{N_{R} 1} & \mathrm{D}_{m}^{N_{R} 2} & \ldots & \mathrm{D}_{m}^{N_{R} N_{T}}
\end{array}\right]
$$

and the noise vector $\tilde{\mathbf{n}}_{m}$ is complex Gaussian with covariance matrix $\mathbf{K}_{\tilde{\mathbf{n}}}=$ $\sigma_{n}^{2} \mathbf{I}_{N_{R}}$

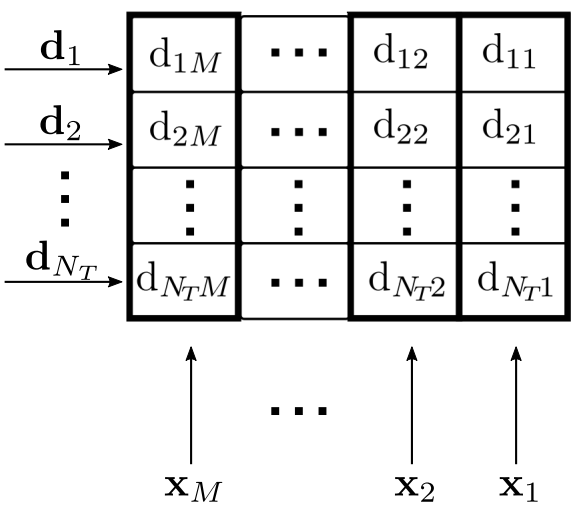

Figure 2.5: Data-matrix $\mathcal{D}$ for SU-MIMO-OFDM system (non-precoded case).

Furthermore, if the channel vectors $\left\{\mathbf{h}_{i j}\right\}$ are i.i.d, then the entries of $\mathbb{D}_{m}$ are i.i.d complex random variables. Therefore, the receiver can retrieve the data matrix $\mathcal{D}$ by performing $M$ independent MIMO-like detections in flat-fading channel, using for example ZF or MMSE linear detectors, each requiring the inversion of a matrix with dimension much smaller than that of $\mathbf{H}$ in (2-4).

\subsubsection{2}

\section{Precoded case}

The main advantage of precoding in single-user scenario is to simplify the detection process. The model, in this case, is similar to the one proposed 
in (2-5). In this case, $\mathbf{x}_{m}=\mathbf{P}_{m} \mathbf{s}_{m}$ where $\mathbf{P}_{m} \in \mathbb{C}^{N_{T} \times N_{R}}$ is the precoding matrix and $\mathbf{s}_{m} \in \mathbb{C}^{N_{R} \times 1}$ is the symbol vector. Then the received vector is given by

$$
\mathbf{r}_{m}=\mathbb{D}_{m} \mathbf{P}_{m} \mathbf{s}_{m}+\tilde{\mathbf{n}}_{m} \quad ; \quad m=1,2, . ., M .
$$

Here the transmission matrix is given by $T_{P}=\mathcal{D}_{P} \mathbf{W}_{M}^{*}$, where

$$
\mathcal{D}_{P}=\left[\begin{array}{llll}
\mathbf{P}_{M} \mathbf{s}_{M} & \ldots & \mathbf{P}_{2} \mathbf{s}_{2} & \mathbf{P}_{1} \mathbf{s}_{1}
\end{array}\right]
$$

\subsection{2}

\section{Multi-User MIMO-OFDM}

This section introduces the system model used in multi-user MIMOOFDM systems. We only refer to the link from the Base Station to the users (downlink) because it is the most challenging one [19].

Figure 2.6 shows a typical multi-user MIMO-OFDM scenario, where the BS simultaneously transmits to $K$ users. The data are processed using the same procedure explained above (SU-MIMO-OFDM precoded-case), but in this case the system consists of $K$ blocks similar to $\mathcal{D}_{P}$ shown in (2-8). In this case, the transmission matrix is given by

$$
T_{P}^{M U}=\sum_{k=1}^{K} \mathcal{D}_{P}^{k} \mathbf{W}_{M}^{*}
$$

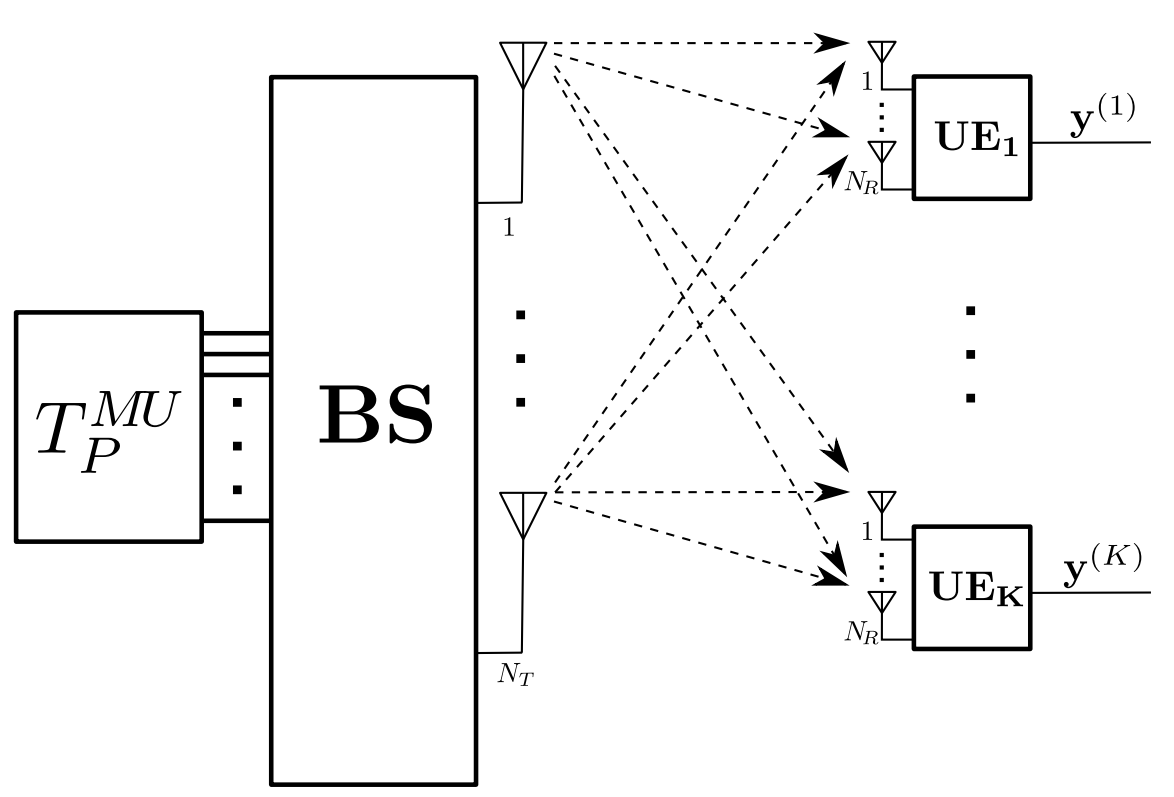

Figure 2.6: Block diagram of the baseband discrete model of a MU-MIMO-OFDM system.

Using the model with $M$ detections introduced in the previous sections, the received signal at the $m$-th detection can be modeled as: 


$$
\mathbf{r}_{m}=\left[\begin{array}{c}
\mathbf{r}_{m}^{(1)} \\
\mathbf{r}_{m}^{(2)} \\
\vdots \\
\mathbf{r}_{m}^{(K)}
\end{array}\right]=\left[\begin{array}{c}
\mathbb{D}_{m}^{(1)} \\
\mathbb{D}_{m}^{(2)} \\
\vdots \\
\mathbb{D}_{m}^{(K)}
\end{array}\right]\left[\begin{array}{llll}
\mathbf{P}_{m}^{(1)} & \mathbf{P}_{m}^{(2)} & \ldots & \mathbf{P}_{m}^{(K)}
\end{array}\right]\left[\begin{array}{c}
\mathbf{s}_{m}^{(1)} \\
\mathbf{s}_{m}^{(2)} \\
\vdots \\
\mathbf{s}_{m}^{(K)}
\end{array}\right]+\left[\begin{array}{c}
\tilde{\mathbf{n}}_{m}^{(1)} \\
\tilde{\mathbf{n}}_{m}^{(2)} \\
\vdots \\
\tilde{\mathbf{n}}_{m}^{(K)}
\end{array}\right]
$$

or

$$
\mathbf{r}_{m}=\mathfrak{D}_{m} \mathfrak{X}_{m}+\mathfrak{N}_{m},
$$

with $\mathfrak{D}_{m}=\left[\begin{array}{llll}\mathbb{D}_{m}^{(1)^{T}} & \mathbb{D}_{m}^{(2)^{T}} \ldots & \mathbb{D}_{m}^{(K)^{T}}\end{array}\right]^{T}, \mathfrak{X}_{m}=\mathfrak{P}_{m} \mathfrak{s}_{m}$, with $\mathfrak{P}_{m}=$ $\left[\begin{array}{llll}\mathbf{P}_{m}^{(1)} & \mathbf{P}_{m}^{(2)} & \ldots & \mathbf{P}_{m}^{(K)}\end{array}\right], \quad \mathbf{s}_{m}=\left[\begin{array}{llll}\mathbf{s}_{m}^{(1)^{T}} & \mathbf{s}_{m}^{(2)^{T}} & \ldots & \mathbf{s}_{m}^{(K)^{T}}\end{array}\right]^{T}$ and $\mathfrak{N}_{m}=$ $\left[\begin{array}{llll}\tilde{\mathbf{n}}_{m}^{(1)^{T}} & \tilde{\mathbf{n}}_{m}^{(2)^{T}} & \ldots & \tilde{\mathbf{n}}_{m}^{(K)^{T}}\end{array}\right]^{T}$. The received signal for each user can be expressed as

$$
\mathbf{r}_{m}^{(k)}=\mathbb{D}_{m}^{(k)} \mathbf{P}_{m}^{(k)} \mathbf{s}_{m}^{(k)}+\mathbb{D}_{m}^{(k)} \sum_{\substack{j=1 \\ j \neq k}}^{K} \mathbf{P}_{m}^{(j)} \mathbf{s}_{m}^{(j)}+\tilde{\mathbf{n}}_{m}^{(k)} \quad ; \quad m=1,2, . ., M
$$

where matrix $\mathbb{D}_{m}^{(k)}$ contains the $N_{R} N_{T}$ elements of the channel impulse response between the BS and the user $k$ at the $m$-th detection, the term $\sum_{\substack{j=1 \\ j \neq k}}^{K} \mathbf{P}_{m}^{(j)} \mathbf{s}_{m}^{(j)}$ represents the interference caused by other users and $\tilde{\mathbf{n}}_{m}^{(k)}$ is the noise vector.

\section{2}

\section{Mobile radio channel characterization}

The mobile radio channel has a direct effect on the performance of wireless communication systems, and it is often difficult to make an accurate analysis of its behavior. That is why its understanding will influence the development of high performance and bandwidth-efficient wireless transmission technologies [20].

The analysis of the mobile radio channel must be done depending on the kind of the environment. For instance, in densely built-up areas, such as cities, the transmitted electromagnetic waves mostly do not arrive at the antenna of the receiver over the direct path. On the other hand, a predominant line-ofsight component often exists as part of the received signal in rural areas [21]. Some phenomena can be more prominent from one scenario to another, due to the environment characteristics.

The three physical phenomena that govern radio waves in terms of propagation mechanisms are reflection, diffraction, and scattering [22], [23].

Reflection is the physical phenomenon that occurs when a propagating electromagnetic wave hits an object with very large dimensions compared to the wavelength. Thus, the signal is reflected back to the transmitter rather 
than being passed all the way along the path to the receiver.

Diffraction makes possible waves to propagate around the curved surface of the earth, beyond the line-of-sight and behind obstructions, but it introduces an additional loss. The received field strength decreases rapidly as a receiver moves deeper into the obstructed region [24]. Following the Huygens principle, points on a wavefront can be considered as point sources, and all of them produce secondary wavelets. The combination of these secondary wavelets produces a new wavefront in the direction of propagation. Diffraction is caused by the propagation of secondary wavelets into a shadowed region.

Scattering forces electromagnetic waves to deviate from a straight path by one or more local obstacles, with small dimensions, compared to the wavelength. The interaction between these waves causes multi-path fading at a given location because their phases are such that sometimes they add, and sometimes they subtract (fade) [24]. This is the reason why the received signal level is often different than what is expected using only reflection and diffraction models. Foliage, street signs, and lampposts are examples of common objects that induce scattering, and they are referred to as the scatters.

The variations of the electromagnetic field magnitude that arise from of all these propagation phenomena are called fading. The modelling of this random process is very important to the communication system.

When considering wireless mobile communications, the environment through which the information signal travels is permanently varying, either by changing the position of the receiver and/or transmitter, or by changing the spatial configuration of the constituting elements of the environment. In this radio-mobile channel communication scenario, fading phenomena can be broadly classified into two types: large-scale fading and small-scale fading.

\subsection{1}

\section{Large-scale fading}

Large-scale fading occurs as the mobile moves through a large distance. It is caused by path loss of signal as a function of distance and shadowing by prominent terrain contours such as buildings, hills, billboards, and vegetation. The statistics of large-scale fading provide a way of computing an estimate of path loss as a function of distance [22]. This is often described in terms of a mean-path loss and a log-normally distributed variation about the mean.

Different models describe this phenomenon, such as the Free-Space Propagation Model, the Okumura/Hata Model, the IEEE 802.16d Model, among others.

The Free-Space Propagation Model is usually used to predict the received 
signal strength in the line-of-sight (LOS) environment where there is no obstacle between the transmitter and receiver.

The Okumura/Hata model was developed using iterative experiments conducted over some time by measuring data in a specific area. It is more accurate in a more realistic scenario (considering the terrain profile). Hence, this model is commonly used as a path loss model to estimate the coverage radius.

IEEE 802.16d model is based on the log-normal shadowing path loss model. This standard consists of three different types of models (Type A, B, and $\mathrm{C}$ ) were defined, depending on the density of obstruction between the transmitter and receiver (in terms of tree densities) in a macro-cell suburban area $[20]$.

\subsection{2}

\section{Small-scale fading}

Small-scale fading models (also known as microscopic fading models) characterize the sudden changes in signal amplitude and phase due to the constructive and destructive interference of multiple signal paths (multi-paths) when the mobile station moves short distances (as short as a half-wavelength) [22]. In other words, the variation of the received signal level is related to the relative phases among the number of signals reflected from the local scatters.

Small-scale fading is called Rayleigh fading if the number of reflective paths is large enough and if there is no dominant component (there is no LOS signal component). If there is a direct (possibly a LOS) path present between transmitter and receiver, the signal envelope is no longer Rayleigh, and the distribution of the signal amplitude is Ricean [24]. There are three types of microscopic fading [25]:

1. Doppler spread - Time selective fading

Depending on the extent of the Doppler spread, the received signal undergoes fast or slow fading. Time selective fading can be characterized by the coherence time, $T_{c}$. This parameter is typically defined as the time lag for which the signal autocorrelation coefficient reduces to $0.7[25]$.

When $T_{c}$ is higher than the symbol period $\left(T_{s}\right)$ (i.e., $T_{c}>T_{s}$ ), the state of the channel will be constant in the symbol duration, and then the pulse distortion will not occur. This is known as slow fading. In a fast fading channel, $T_{c}$ is smaller than the symbol period (i.e., $T_{c}<T_{s}$ ), and thus, the channel impulse response quickly varies 
within the symbol period. Variation in the time domain is closely related to the movement of the transmitter or receiver, which incurs a spread in the frequency domain, known as the Doppler shift $f_{m}$. The coherence time is inversely proportional to the Doppler spread and can be approximated as

$$
T_{c}=\frac{1}{f_{m}}
$$

2. Delay spread - Frequency selective fading

Delay spread causes frequency selective fading as the channel acts like a tapped delay line filter. Frequency selective fading can be characterized in terms of coherence bandwidth $W_{c}$, which is the frequency lag for which the channel's autocorrelation coefficient reduces to 0.7 [25]. The coherence bandwidth is inversely proportional to the delay spread and is a measure of the channel's frequency selectivity. Thus, $W_{c}$ can be obtained from

$$
W_{c}=\frac{1}{T_{d}},
$$

where $T_{d}$ is the delay spread, that is, the standard deviation of the arrival time of all multipaths, weighted by their powers.

When the coherence bandwidth is comparable with or less than the signal bandwidth, the channel is said to be frequency selective. Otherwise, a non-frequency selective or flat-fading channel refers to when $W_{c}$ is higher than the signal bandwidth. In other words, the frequency components in the received signal undergo the same attenuation and phase shift.

3. Angle spread - Space selective fading

Angle spread causes space selective fading, which means that signal amplitude depends on the spatial location of the antenna. Space selective fading is characterized by the coherent distance $D_{c}$, which is the spatial separation for which the autocorrelation coefficient of the spatial fading drops to 0.7 [25]. The coherence distance is inversely proportional to the angle spread (the larger the angle spread, the shorter the coherence distance). 


\section{3}

\section{Detection Techniques}

Linear detectors are used to mitigate the effects of the channel, through the use of a linear filter at the receiver [25]. The desired signal at the receiver is recovered using the equalization process, followed by a decision on the transmitted symbol. The equalization matrix $G$ is chosen depending on the channel. ZF and MMSE are examples of the most common equalizers used in MIMO-OFDM systems. The complexity of linear detectors in the MIMOOFDM system shown here is dominated by the inversion or factorization of a matrix with $N_{R} \times N_{T}$ dimension.

\subsection{1}

\section{Zero-Forcing Detection, single-user}

Linear detectors based on the principle of Zero-Forcing (ZF) eliminates interference among the spatial layers entirely by filtering the received signal with an inverse of the channel matrix. This method does not consider the effects of noise [26], and therefore the noise may be enhanced in the process of eliminating interference. Thus, the bit-error rate (BER) performance of the ZF detector can be highly reduced. ZF equalizers assume that the number of receiving antennas is greater than the number of transmitting antennas and the equalization matrix, $\mathbf{G}_{m}^{Z F} \in \mathbb{C}^{N_{T} \times N_{R}}$ can be obtained from

$$
\mathbf{G}_{m}^{Z F}=\left(\mathbb{D}_{m}\right)^{\dagger}=\left(\mathbb{D}_{m}^{H} \mathbb{D}_{m}\right)^{-1} \mathbb{D}_{m}^{H}
$$

where $\mathbb{D}_{m}$ represents the channel matrix for the $m$-th detection. Then the estimated vector can be expressed as

$$
\begin{aligned}
\hat{\mathbf{x}}_{m}^{Z F} & =\mathbf{G}_{m}^{Z F} \mathbf{r}_{m} \\
& =\left(\mathbb{D}_{m}^{H} \mathbb{D}_{m}\right)^{-1}\left(\mathbb{D}_{m}^{H} \mathbb{D}_{m} \mathbf{x}_{m}+\mathbb{D}_{m}^{H} \tilde{\mathbf{n}}_{m}\right) \\
& =\mathbf{x}_{m}+\tilde{\mathbf{n}}_{m}^{Z F}
\end{aligned}
$$

where $\tilde{\mathbf{n}}_{m}^{Z F}=\left(\mathbb{D}_{m}^{H} \mathbb{D}_{m}\right)^{-1} \mathbb{D}_{m}^{H} \tilde{\mathbf{n}}_{m}$. Thus, the detection of the data vector is then simplified to element-wise minimum distance detection:

$$
\hat{\mathbf{x}}_{m}=\mathcal{Q}\left(\hat{\mathbf{x}}_{m}^{Z F}\right),
$$

where $\mathcal{Q}(\mathbf{x})=\left[\begin{array}{llll}\mathcal{Q}\left(x_{1}\right) & \mathcal{Q}\left(x_{2}\right) & \ldots & \mathcal{Q}\left(x_{m}\right)\end{array}\right]^{T}$ and $\mathcal{Q}(x)$ returns the element of $\mathcal{M}$ closest to $x$. 


\subsection{2}

\section{Minimum Mean Square Error Detector, single-user}

MMSE detector offers improvements over the ZF equalization, especially related to the poor performance of that equalizer in low SNR regimes. MMSE achieves a trade-off between the noise level and the interference at the receiver, by maximizing the signal-to-interference-plus-noise ratio (SINR). The MMSE filter $\mathbf{G}_{m}^{M M S E}$ can be computed by minimizing the mean square error (MSE) $[27]$ as

$$
\begin{aligned}
\mathbf{G}_{m}^{M M S E} & =\underset{\mathbf{G}}{\arg \min } \mathbb{E}\left\{\left\|\mathbf{x}_{m}-\mathbf{G r}_{m}\right\|^{2}\right\} \\
& =\mathbb{E}\left\{\mathbf{x}_{m} \mathbf{r}_{m}^{H}\right\}\left(\mathbb{E}\left\{\mathbf{r}_{m} \mathbf{r}_{m}^{H}\right\}\right)^{-1} \\
& =\mathbb{D}_{m}^{H}\left(\mathbb{D}_{m} \mathbb{D}_{m}^{H}+\frac{\sigma_{n}^{2}}{E_{s}} \mathbf{I}_{N_{R}}\right)^{-1} \\
& =\left(\mathbb{D}_{m}^{H} \mathbb{D}_{m}+\frac{\sigma_{n}^{2}}{E_{s}} \mathbf{I}_{N_{T}}\right)^{-1} \mathbb{D}_{m}^{H}
\end{aligned}
$$

The estimated symbol vector is given by

$$
\begin{aligned}
\hat{\mathbf{x}}_{m}^{M M S E} & =\mathbf{G}_{m}^{M M S E} \mathbf{r}_{m} \\
& =\mathbf{G}_{m}^{M M S E} \mathbb{D}_{m} \mathbf{x}_{m}+\mathbf{G}_{m}^{M M S E} \tilde{\mathbf{n}}_{m}
\end{aligned}
$$

and then $\mathbf{x}_{m}$ can be obtained from

$$
\hat{\mathbf{x}}_{m}=\mathcal{Q}\left(\hat{\mathbf{x}}_{m}^{M M S E}\right) .
$$

\section{4}

\section{Precoding for multi-user system}

Precoding is a technique that processes the signal before transmission. In a single-user scenario, precoding techniques are commonly used to simplify the process at the receiver (often to avoid the equalization procedure). However, in a multi-user scenario, due to the need for separating the data vector for each user (i.e. to mitigate multi-user interference - MUI), precoders are a fundamental step before transmitting the data signal.

Precoding design varies depending on the types of channel state information (CSI) and the performance criterion. The CSI necessary for precoding is obtained from the users through estimation techniques, using feedback channel in Frequency Division Duplexing (FDD) or reciprocity in Time Division Duplexing (TDD) [28]. In FDD systems, uplink and downlink use different fre- 
quency bands, allowing full duplex transmission. FDD usually does not have channel reciprocity between uplink and downlink, so CSI must be estimated at the receiver side and then, fed back to the transmitter side [20]. Additional resources are necessary for transmitting the feedback information, and the greater the number of antennas, the greater the requirement of feedback information. On the other hand, in TDD system, different time intervals are used to allocate the information, and the same frequency band is used for both downlink and uplink. Here, the channel reciprocity is exploited as a mechanism used to get CSI, and based on this assumption, only CSI for the uplink needs to be estimated. TDD operation is independent of the number of BS antennas. Here, the main disadvantage is that the time required to acquire CSI depends on the number of users [28].

Precoding techniques can be linear or non-linear. A linear precoder (also simply called Beamforming) essentially functions as a multimode beamformer, optimally matching the input signal on one side to the channel on the other side [29]. Due to their simplicity, only linear precoders have been used in this work.

\subsection{1}

\section{Zero-Forcing}

Zero-Forcing is a linear precoding technique with reasonable computational complexity. It achieves a good performance at high signal-to-noise ratio (SNR) values. However, it performs far from optimal in the noise limited regime [30]. When CSI is available on the transmission side, this method becomes a useful solution because it cancels the multi-user interference term, from (212 ), i.e. $\mathbb{D}_{m}^{(k)} \mathbf{P}_{m}^{(j)}=0, \forall k \neq j$. Considering the signal model in (2-11) the ZF precoder, $\mathfrak{P}_{m}$, is designed in such a way that

$$
\mathfrak{D}_{m} \mathfrak{P}_{m}=\alpha_{Z F} \mathbf{I}_{K N_{R}} .
$$

The scalar factor $\alpha_{Z F} \in \mathbb{R}_{+}$has to be selected in such a way that the transmitted energy does not exceed the average energy available at the transmitter given by (see appendix A)

$$
\begin{aligned}
E_{T} & =\frac{1}{M} \sum_{m=1}^{M} \mathbb{E}\left\{\left\|\mathfrak{X}_{m}\right\|^{2}\right\} \\
& =\frac{1}{M} \sum_{m=1}^{M} \mathbb{E}\left\{\left\|\mathfrak{P}_{m} \mathfrak{s}_{m}\right\|^{2}\right\} .
\end{aligned}
$$

The ZF precoder is then given by 


$$
\mathfrak{P}_{m}^{Z F}=\alpha_{Z F} \mathfrak{D}_{m}^{H}\left(\mathfrak{D}_{m} \mathfrak{D}_{m}^{H}\right)^{-1},
$$

and $\alpha_{Z F}$ can be obtained from $(2-22)$

where

$$
\alpha_{Z F}=\sqrt{\frac{E_{T}}{\gamma_{Z F}}},
$$

$$
\gamma_{Z F}=\frac{1}{M} \sum_{m=1}^{M} \operatorname{Tr}\left(\left(\mathfrak{D}_{m} \mathfrak{D}_{m}^{H}\right)^{-1} \mathfrak{R}_{\mathbf{s}_{m}}\right)
$$

and $\boldsymbol{R}_{\mathbf{s}_{m}}$ is the autocorrelation matrix of $\mathfrak{s}_{m}$.

\subsection{2}

\section{Minimum Mean Square Error}

ZF precoder solves the MUI problem by canceling the interference term, as shown in the previous section, but its performance can be poor for low SNR values. The optimal solution to mitigate this problem can be the use of MMSE precoder, because it achieves an optimum trade-off between the SNR and the interference elimination.

Similar to ZF precoder, we can introduce a scalar factor $\alpha_{M M S E} \in \mathbb{R}_{+}$to control the selection of the precoding matrix $\mathfrak{P}_{m}^{M M S E}$ based on the transmitted power. To derive the expression for the MMSE precoder, we have to solve the following optimization problem

$$
\begin{gathered}
\left\{\mathfrak{P}_{1}^{M M S E}, \mathfrak{P}_{2}^{M M S E}, \ldots, \mathfrak{P}_{M}^{M M S E}\right\}=\underset{\left\{\mathfrak{P}_{1}, \mathfrak{P}_{2}, \ldots, \mathfrak{P}_{M}\right\}}{\arg \min } \sum_{m=1}^{M} \mathbb{E}\left\{\left\|\mathfrak{s}_{m}-\left(\alpha_{M M S E}\right)^{-1} \mathbf{r}_{m}\right\|^{2}\right\} \\
\text { s.t.: } \frac{1}{M} \sum_{m=1}^{M} \mathbb{E}\left[\left\|\mathfrak{P}_{m} \mathfrak{s}_{m}\right\|^{2}\right] \leq E_{T}
\end{gathered}
$$

Using the Lagrangian multiplier method [31], it is possible to find the optimal solutions for $\mathfrak{P}_{m}^{M M S E}, m=1,2, \ldots, M$ and $\alpha_{M M S E}$, given by

$$
\begin{aligned}
\mathfrak{P}_{m}^{M M S E} & =\alpha_{M M S E} \mathfrak{D}_{m}^{H}\left(\mathfrak{D}_{m} \mathfrak{D}_{m}^{H}+\frac{\frac{1}{M} \sum_{m=1}^{M} \operatorname{Tr}\left(\mathfrak{R}_{\mathbf{n}_{m}}\right)}{E_{T}} \mathbf{I}_{K N_{R}}\right)^{-1} \\
\alpha_{M M S E} & =\sqrt{\frac{E_{T}}{\gamma_{M M S E}}}
\end{aligned}
$$

where

$$
\gamma_{M M S E}=\frac{1}{M} \sum_{m=1}^{M} \operatorname{Tr}\left(\mathbf{F}_{m}^{H} \mathfrak{D}_{m} \mathfrak{D}_{m}^{H} \mathbf{F}_{m} \mathfrak{R}_{\mathbf{s}_{m}}\right),
$$

and

$$
\mathbf{F}_{m}=\left(\mathfrak{D}_{m} \mathfrak{D}_{m}^{H}+\frac{\frac{1}{M} \sum_{m=1}^{M} \operatorname{Tr}\left(\mathfrak{R}_{\mathbf{n}_{m}}\right)}{E_{T}} \mathbf{I}_{K N_{R}}\right)^{-1}
$$


where $\boldsymbol{R}_{\mathbf{n}_{m}}$ denotes noise covariance matrix of the vector $\mathfrak{N}_{m}$ defined in connection with (2-11). 


\section{3}

\section{Transmit Antennas Selection}

The main advantages of MIMO systems are the improvement in the performance and capacity of the system without additional transmission energy or bandwidth extension. These systems are usually equipped with multiple antennas at both transmitter and receiver. That means an increase in complexity due to a greater number of RF chains composed by amplifiers, converters, etc. One feasible solution to overcome this drawback is the antenna selection technique.

Suppose that the transmitter shown in Figure 3.1 is equipped with a reduced number, $N_{R F}$, of RF chains, and the number of available transmitting antennas is higher than $N_{R F}$, i.e. $N_{T}>N_{R F}$. The objective here is to select the best subset of $N_{R F}$ transmit antennas according to the current characteristics of the transmission channels.

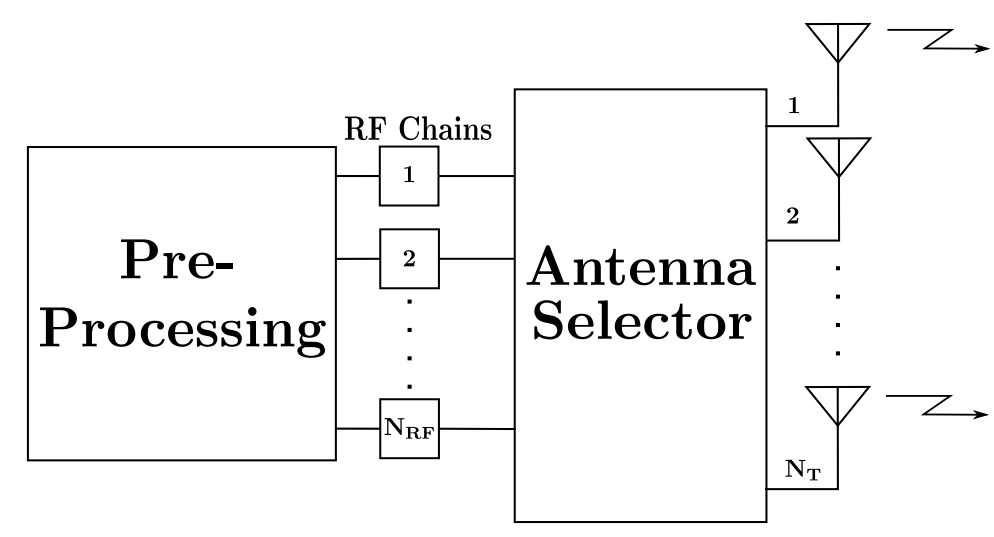

Figure 3.1: MIMO-OFDM system with antenna selection at the transmitter.

In this chapter, we present a general model to describe the transmit antennas selection for SU-MIMO-OFDM (non-precoded) and MU-MIMOOFDM (precoded) systems. Transmit antenna selection strategies are proposed for these two models. Simulation results describing the BER performance of the system are presented, employing ZF and MMSE equalizers for SU-MIMOOFDM case, as well as ZF and MMSE as precoders in the MU-MIMO-OFDM scenario. 


\section{1}

\section{SU-MIMO-OFDM system (non-precoded)}

\subsection{1}

\section{Transmit Antenna Selection Model for SU-MIMO-OFDM systems}

As in [11],[32], let $\mathbf{p} \in\{1,0\}^{N_{T}}$ denote a vector that indicates the subset of antennas that are activated, from the total set of $N_{T}$ antennas. For instance, let $N_{T}=7$ and $N_{R F}=4$, pattern $\mathbf{p}=\left[\begin{array}{lllllll}1 & 0 & 1 & 0 & 1 & 0 & 1\end{array}\right]^{T}$ indicates that antennas with odd indexes are selected and the remaining are deactivated. The corresponding effective channel matrix, $\mathbb{D}_{m}(\mathbf{p}) \in \mathbb{C}^{N_{R} \times N_{R F}}$ is obtained by selecting the columns indexed by $\mathbf{p}$. Thus,

$$
\mathbb{D}_{m}(\mathbf{p})=\mathbb{D}_{m} \mathbf{U}(\mathbf{p})
$$

where $\mathbf{U}(\mathbf{p}) \in \mathbb{C}^{N_{T} \times N_{R F}}$ is the matrix obtained from $\mathbf{I}_{N_{T}}$ eliminating its $i$-th column if the $i$-th component of vector $\mathbf{p}$ is zero. It then results that $\mathbf{U}(\mathbf{p}) \mathbf{U}^{T}(\mathbf{p})=\operatorname{diag}(\mathbf{p})=\mathbf{B}(\mathbf{p})$.

Let $C_{t}$ be the set of possible $\mathbf{p}$ patterns indicating $N_{R F}$-selected out of $N_{T}$ antennas, then the total number of possible combinations is given by

$$
\left|C_{t}\right|=\left(\begin{array}{c}
N_{T} \\
N_{R F}
\end{array}\right)=\frac{N_{T} !}{\left(N_{T}-N_{R F}\right) ! N_{R F} !} .
$$

\subsection{2}

\section{Mutual Information Approach (MIA)}

This method is based on the maximization of the mutual information between the transmitted and received signals [33]. Since in the model related to (2-5), all $M$ detections are independent, the total mutual information $\mathbf{I}$, is the sum of the individual mutual informations. That is, for a given $\mathbf{p}$ vector, the transmitter computes the mutual information [34], as

$$
\mathbf{I}(\mathbf{p})=\sum_{m=1}^{M} \log _{2} \operatorname{det}\left[\frac{E_{s}}{\sigma_{n}^{2}} \mathbb{D}_{m} \mathbf{B}(\mathbf{p}) \mathbb{D}_{m}^{H}+\mathbf{I}_{N_{R}}\right] .
$$

where $E_{s}$ is the average information symbol energy given by (see appendix B)

$$
E_{s}=\frac{E_{T}}{N_{R F}} .
$$

The antenna selection is indicated by the pattern $\mathbf{p}_{*}$ given by

$$
\mathbf{p}_{*}=\underset{\mathbf{p} \in \mathrm{C}_{t}}{\arg \max } \mathbf{I}(\mathbf{p})
$$




\subsection{3}

\section{Mean Square Error (MSE) Minimizing Approach}

As shown in the previous section, the mutual information can be used to choose a convenient subset of antennas to transmit. This approach is considered as a general method to do antenna selection and doesn't take advantage of the type of equalizer used in the detection. Another more specific method is proposed. This approach is based on the minimization of the MSE (after equalization) and therefore, takes into account the particular expression for the current equalizer.

\subsubsection{1}

\section{MSE Minimizing approach in ZF-equalized receiver}

From (2-16) it is evident that after ZF equalization the estimated vector $\hat{\mathbf{x}}_{m}^{Z F}$ is affected by the equalization matrix $\mathbf{G}_{m}^{Z F}$ only due to its effect in the noise vector. The MSE, in this case, is given by

$$
\begin{aligned}
M S E_{Z F} & =\sum_{m=1}^{M} \mathbb{E}\left\{\left\|\hat{\mathbf{x}}_{m}^{Z F}-\mathbf{x}_{m}\right\|^{2}\right\} \\
& =\sum_{m=1}^{M} \mathbb{E}\left\{\left\|\mathbf{G}_{m}^{Z F} \tilde{\mathbf{n}}_{m}\right\|^{2}\right\} \\
& =\sum_{m=1}^{M} \operatorname{Tr}\left\{\mathbb{E}\left[\mathbf{G}_{m}^{Z F} \tilde{\mathbf{n}}_{m} \tilde{\mathbf{n}}_{m}^{H} \mathbf{G}_{m}^{Z F^{H}}\right]\right\} \\
& =\sigma_{n}^{2} \sum_{m=1}^{M} \operatorname{Tr}\left\{\mathbf{G}_{m}^{Z F} \mathbf{G}_{m}^{Z F^{H}}\right\},
\end{aligned}
$$

and substituting the matrix $\mathbf{G}_{m}^{Z F}$ by the expression obtained from (2-15), we can express the MSE as

$$
M S E_{Z F}=\sigma_{n}^{2} \sum_{m=1}^{M} \operatorname{Tr}\left\{\left(\mathbb{D}_{m}^{H} \mathbb{D}_{m}\right)^{-1}\right\}
$$

Therefore, the antenna selection, indicated by the pattern $\mathbf{p}_{*}$, can be calculated as

$$
\mathbf{p}_{*}=\underset{\mathbf{p}}{\arg \min } \sigma_{n}^{2} \sum_{m=1}^{M} \operatorname{Tr}\left\{\left(\mathbb{D}_{m}^{H}(\mathbf{p}) \mathbb{D}_{m}(\mathbf{p})\right)^{-1}\right\}
$$




\subsubsection{2}

\section{MSE Minimizing approach in MMSE-equalized receiver}

The expression for MSE in the MMSE-equalized receiver is a bit more complicated than the one shown in the previous section because the MMSE equalizer does not eliminate the channel matrix. The MSE in this case is given by

$$
M S E_{M M S E}=\sum_{m=1}^{M} \mathbb{E}\left\{\left\|\hat{\mathbf{x}}_{m}^{M M S E}-\mathbf{x}_{m}\right\|^{2}\right\}
$$

Using (2-18) and (2-19), we can show that the solution for (3-8) can be written as

$$
M S E_{M M S E}=\sigma_{n}^{2} \sum_{m=1}^{M} \operatorname{Tr}\left\{\left(\mathbb{D}_{m}^{H} \mathbb{D}_{m}+\frac{\sigma_{n}^{2}}{E_{s}} \mathbf{I}_{N_{T}}\right)^{-1}\right\} .
$$

The best pattern for the vector $\mathbf{p}$ can be calculated by minimizing the $M S E_{M M S E}$ and is given by

$$
\mathbf{p}_{*}=\underset{\mathbf{p}}{\arg \min } \sigma_{n}^{2} \sum_{m=1}^{M} \operatorname{Tr}\left\{\left(\mathbb{D}_{m}^{H}(\mathbf{p}) \mathbb{D}_{m}(\mathbf{p})+\frac{\sigma_{n}^{2}}{E_{s}} \mathbf{I}_{N_{R F}}\right)^{-1}\right\} .
$$

\subsection{4}

\section{Simulation Results}

In this section, numerical results are presented to evaluate the BER performance in the SU-MIMO-OFDM scenario, using different approaches to select the best subset of antennas to transmit. The curves are obtained after $N_{C H}$ independent realizations of the channel matrix. The coefficients of the adopted discrete impulse response of the channels have the form $h(l)_{i j}=p(l) \alpha_{i j}(l) \quad, i=1,2, . ., N_{R}, j=1,2, \ldots, N_{T}, l=1,2 \ldots, L$ where the $N_{R} \times N_{T} \times L$ random variables $\alpha_{i j}(l)$ are statistically independent, complex Gaussian with zero-mean and variance 1 . The weights have an exponential decay, that is

$$
p(l)=10 \exp \{-l /(L-1)\},
$$

and are further normalized such that

$$
\sum_{l=1}^{L} p^{2}(l)=1
$$

Thus, resulting that $\mathbb{E}\left[\left\|\mathbf{h}_{i j}\right\|^{2}\right]=1$.

The noise vector $\tilde{\mathbf{n}}_{m}$ in (2-5) is a complex zero-mean Gaussian vector with circularly symmetric components and covariance matrix $\mathbf{K}_{\tilde{\mathbf{n}}}=\sigma_{n}^{2} \mathbf{I}_{N_{R}}$. Results are expressed in terms of signal-to-noise ratio 


$$
\mathrm{SNR}_{[\mathrm{dB}]}=10 \log _{10}\left(\frac{E_{T}}{\sigma_{n}^{2}}\right),
$$

and QPSK modulation is assumed. The length of the OFDM blocks is $M=64$.

BER values are estimated via Monte-Carlo method after the transmission of $N_{S Y M}$ information symbols, with a new independent realization of the $N_{R} N_{T}$ random channel vectors $\left\{\mathbf{h}_{i j}\right\}$ generated after the transmission of $N_{D M}$ data matrices $\mathcal{D}$.

\subsubsection{1}

\section{ZF and MMSE for MIA}

Figures 3.2 and 3.3 depict the detection performance for a range of SNR, considering ZF and MMSE equalization, respectively, varying the number of available transmit antennas, while the number of $\mathrm{RF}$ chains and receive antennas is kept fixed. In both cases, detection performance is improved when MIA is employed as the antenna selection strategy. Moreover, the improvement is greater as the number of antennas available at the transmitter increases. For instance, the systems with $N_{T}=10$, in Figures 3.2 and 3.3, require approximately $9.8 \mathrm{~dB}$ and $9.5 \mathrm{~dB}$ less energy, respectively, compared to the case where $N_{T}=2$, when antenna selection is not employed. These results were obtained after transmitting a total of $N_{S Y M}=12.8 \times 10^{7}$ symbols and $N_{D M}=20$ data-matrices for each of $N_{C H}=5 \times 10^{4}$ channel realizations. 


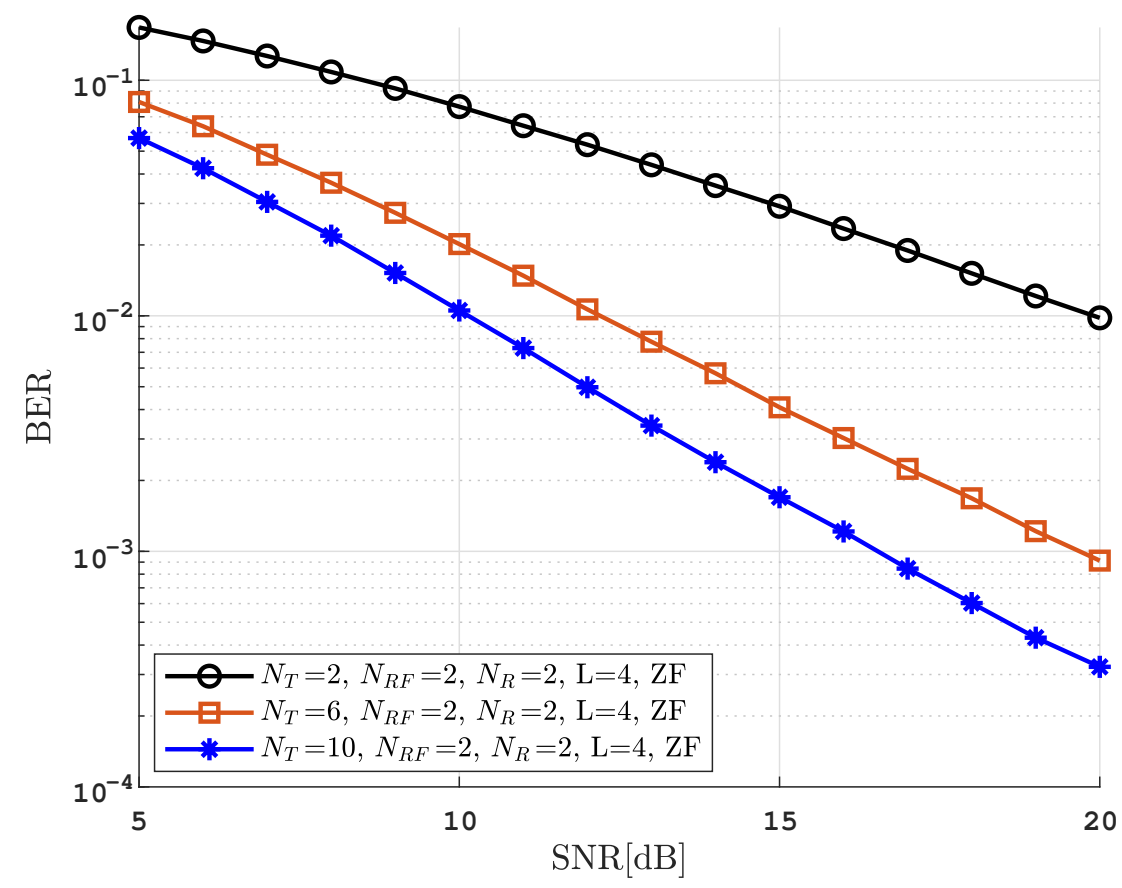

Figure 3.2: BER vs. SNR [dB] for transmit antenna selection using Mutual Information Approach, ZF equalizer, and considering a different number of available antennas at the transmitter.

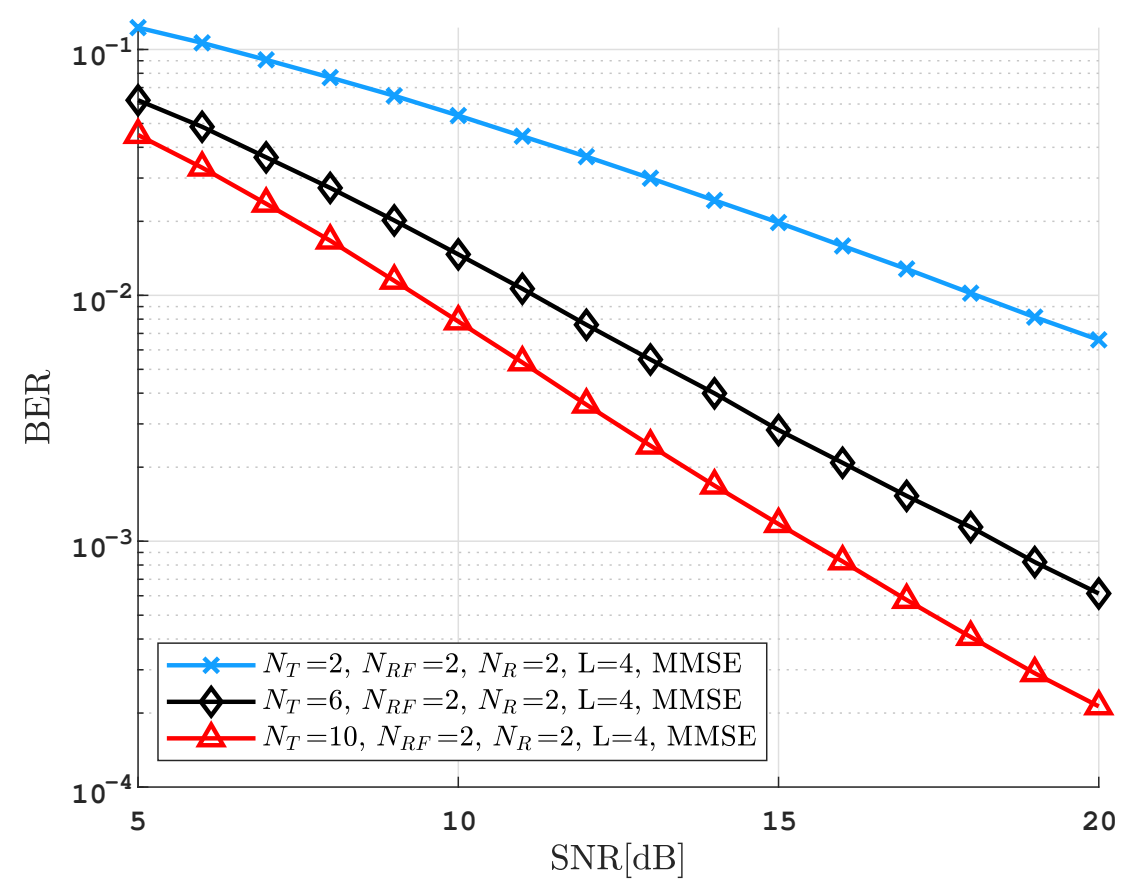

Figure 3.3: BER vs. SNR [dB] for transmit antenna selection using Mutual Information Approach, MMSE equalizer, and considering a different number of available antennas at the transmitter. 


\subsubsection{2}

\section{ZF and MMSE for MSE approach}

Simulation in Figures 3.4 and 3.5 depicts the detection performance for ZF and MMSE equalization, respectively. They were both obtained using the MSE approach as the antenna selection strategy. We can see that the system with $N_{T}=10$, in both cases, requires significantly less energy (a decrease greater than $10 \mathrm{~dB}$ in number) compared to the case where $N_{T}=2$, when no antenna selection strategies are employed. These results were gathered after transmitting a total of $N_{S Y M}=12.8 \times 10^{7}$ symbols and $N_{D M}=20$ datamatrices for each of $N_{C H}=5 \times 10^{4}$ channel realizations.

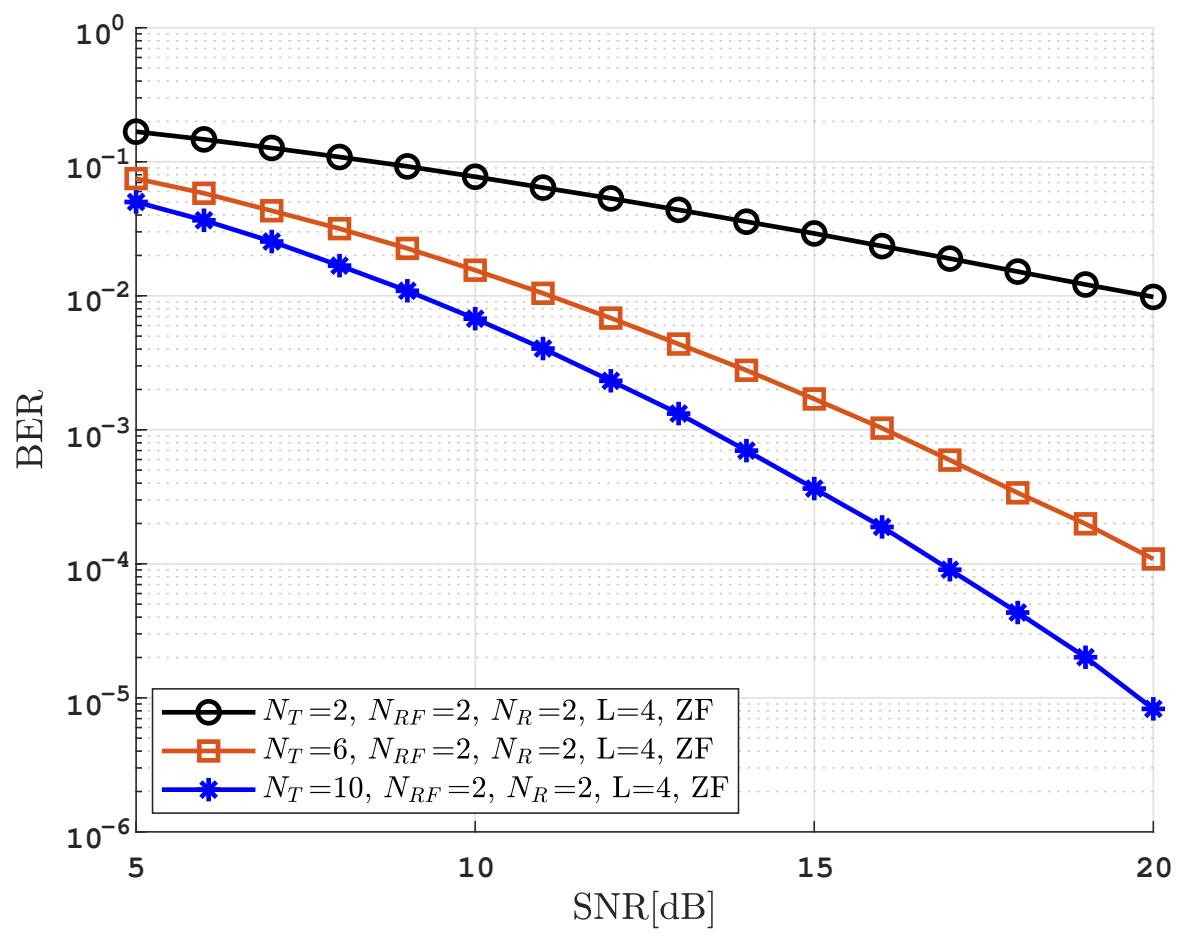

Figure 3.4: BER vs. SNR [dB] for transmit antenna selection using MSE Approach, ZF equalizer, and considering a different number of available antennas at the transmitter. 


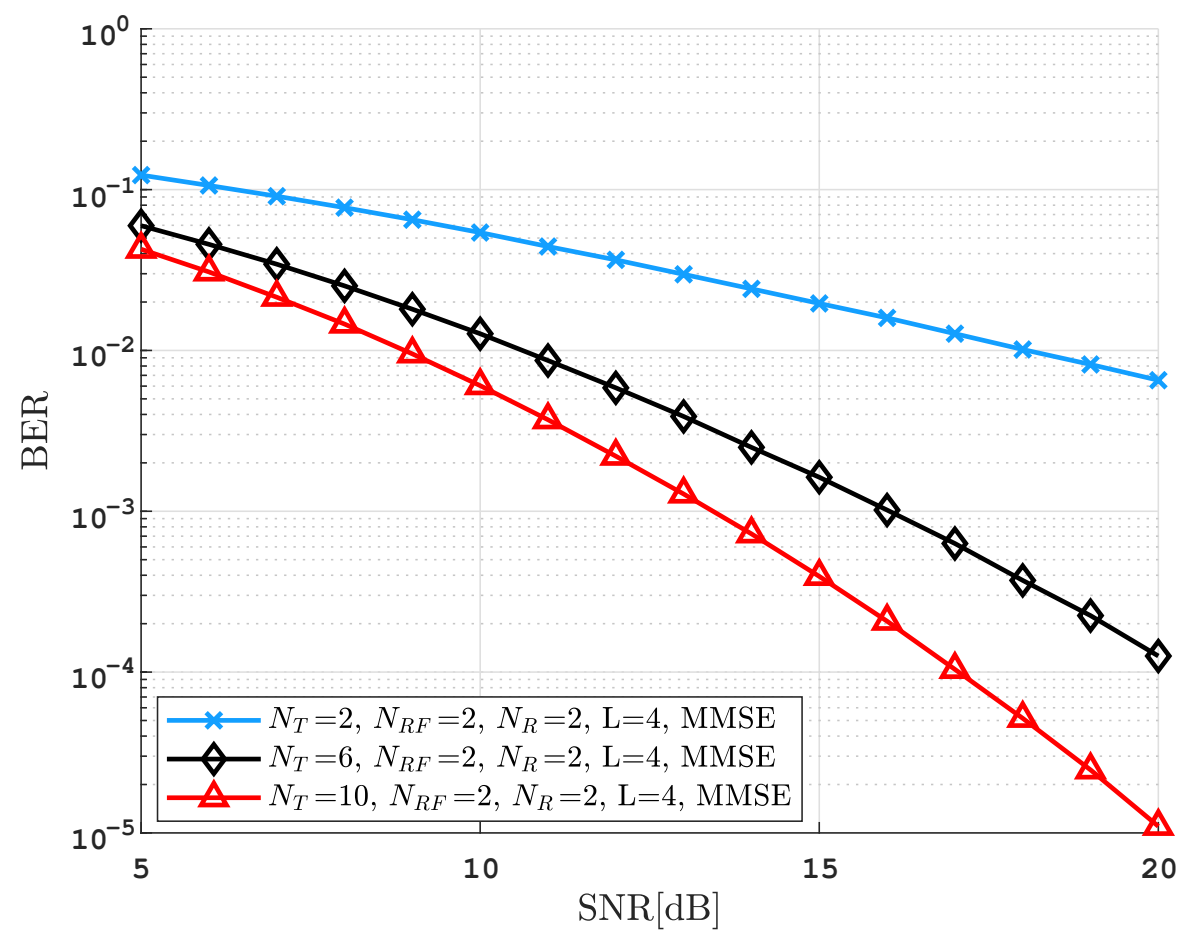

Figure 3.5: BER vs. SNR [dB] for transmit antenna selection using MSE Approach, MMSE equalizer, and considering a different number of available antennas at the transmitter.

\subsubsection{3 \\ Comparisons}

This short section focuses on showing comparisons between the two proposed methods (MIA and MSE) and the random selection (RAN), in terms of system performance.

In Figures 3.6 and 3.7, the improvement of the system when the proposed methods are used is noticeable. The result for random selection is very poor and similar to the performance when no antenna selection method is employed, i.e., $N_{T}=2$ curves in Figures 3.2 and 3.4 for ZF and 3.3 and 3.5 for MMSE. As expected, the performance at higher SNR values using MSE is better than MIA. This occurs because the selection that uses the MSE approach is specific for each equalizer as opposed to the mutual information selection, which does not depend on the kind of equalizer employed, and can be used interchangeably, for the ZF-equalized or the MMSE-equalized system. Doing a numerical analysis, MSE needs approximately $4.8 \mathrm{~dB}$ and $4.1 \mathrm{~dB}$ less energy than MIA, for ZF-equalized and MMSE-equalized systems, respectively, but its complexity is significantly higher due to the matrix inversion operation. 


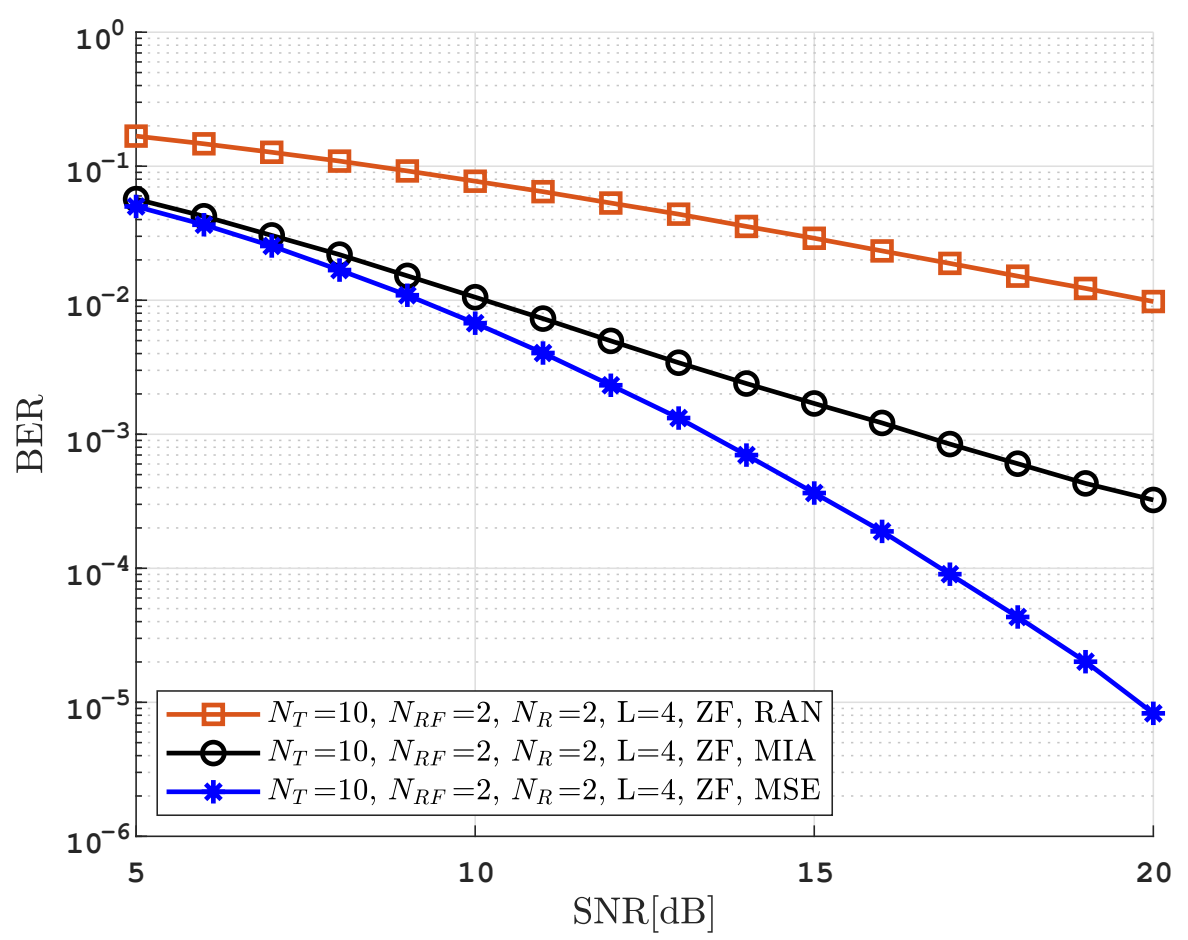

Figure 3.6: BER vs. SNR [dB] for transmit antenna selection using the proposed methods (MIA and MSE) and random selection. Results were obtained employing ZF equalizer.

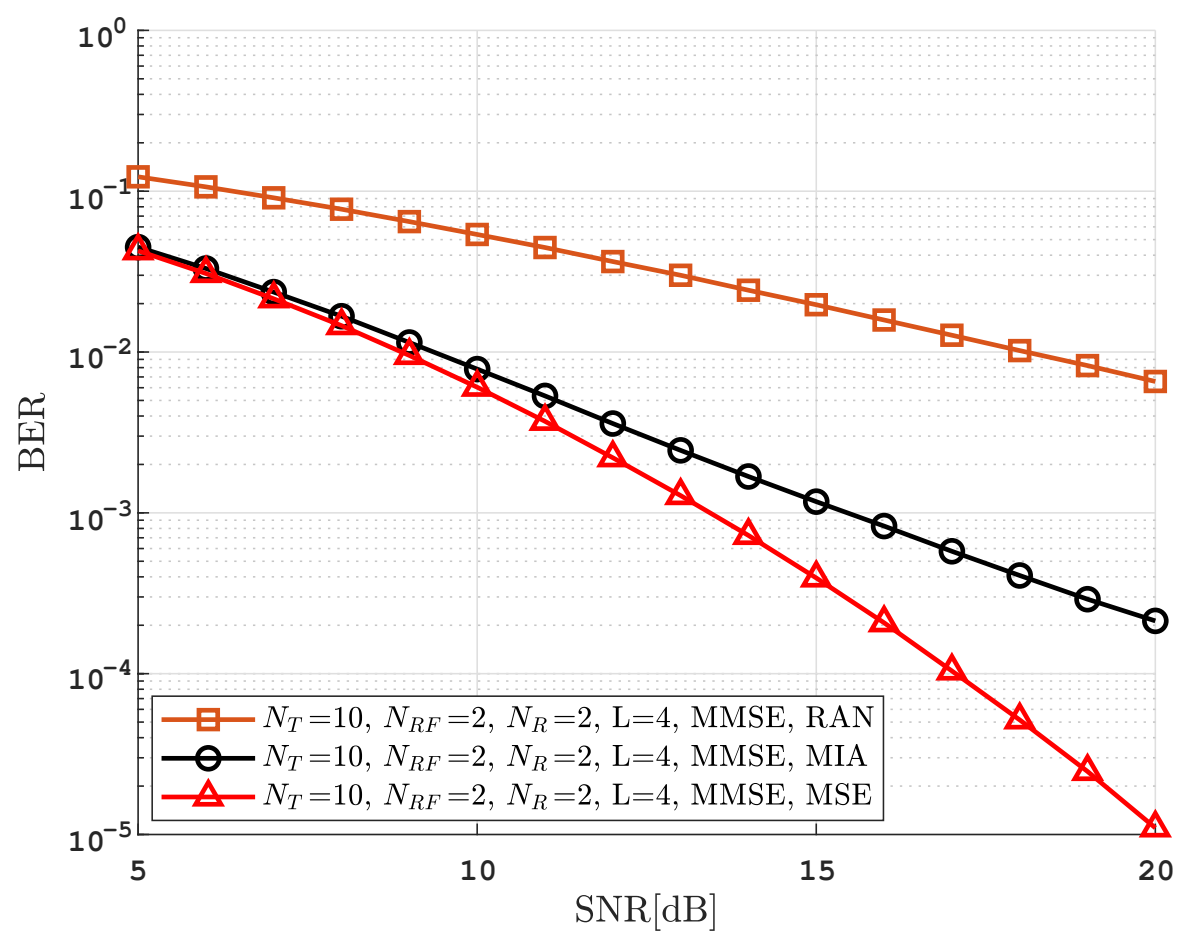

Figure 3.7: BER vs. SNR [dB] for transmit antenna selection using the proposed methods (MIA and MSE) and random selection. Results were obtained employing MMSE equalizer. 


\section{2}

\section{MU-MIMO-OFDM system (precoded)}

\subsection{1}

\section{Transmit Antenna Selection Model for MU-MIMO-OFDM systems}

In MU-MIMO-OFDM scenario, the vector $\mathbf{p}$ is also used to indicate the subset of antennas that are activated in the same way as shown in Section 3.1.1. The corresponding effective channel matrix $\mathfrak{D}_{m}(\mathbf{p}) \in \mathbb{C}^{K N_{R} \times N_{R F}}$, following the system model proposed in (2-11), is obtained by selecting the columns indexed by $\mathbf{p}$. Thus,

$$
\mathfrak{D}_{m}(\mathbf{p})=\mathfrak{D}_{m} \mathbf{U}(\mathbf{p}),
$$

where $\mathbf{U}(\mathbf{p})$ is the matrix obtained from $\mathbf{I}_{N_{T}}$ eliminating its $i$-th column if the $i$-th component of vector $\mathbf{p}$ is zero. It also results that $\mathbf{U}(\mathbf{p}) \mathbf{U}^{T}(\mathbf{p})=$ $\operatorname{diag}(\mathbf{p})=\mathbf{B}(\mathbf{p})$.

\subsection{2}

\section{Transmit Antenna Selection Approach for ZF Precoding}

With antenna selection, expression $(2-11)$ is modified. For a given $\mathbf{p}$ vector, the received vector can be expressed as

$$
\mathbf{r}_{m}=\mathfrak{D}_{m}(\mathbf{p}) \mathfrak{P}_{m}^{Z F}(\mathbf{p}) \mathfrak{s}_{m}+\mathfrak{N}_{m},
$$

where $\mathfrak{P}_{m}^{Z F}(\mathbf{p}) \mathfrak{s}_{m} \in \mathbb{C}^{N_{R F} \times 1}$. For a ZF-precoded system, the precoding matrix $\mathfrak{P}_{m}^{Z F}(\mathbf{p})$ is given by the right pseudo-inverse of $\mathfrak{D}_{m}(\mathbf{p})$ in (2-23), and using this expression in (3-15), the received vector is written as

$$
\mathbf{r}_{m}=\alpha_{Z F}(\mathbf{p}) \mathfrak{s}_{m}+\mathfrak{N}_{m}
$$

where $\alpha_{Z F}(\mathbf{p})$ is obtained from (2-24).

Now, the key point is about finding the optimum subset of $N_{R F}$ transmit antennas. In [35-37], the best solution is found through the channel capacity maximization, while in [38] and [39] a channel-norm-based selection is discussed. From (3-16), we can see that maximizing the value of $\alpha_{Z F}(\mathbf{p})$, we maximize the signal level at the receiver without any other modification in the noise vector, and therefore it maximizes the SNR of the system. The proposed method to choose the best pattern of the vector $\mathbf{p}$ is then calculated as

$$
\begin{aligned}
\mathbf{p}_{*} & =\underset{\mathbf{p} \in \mathrm{C}_{t}}{\arg \max } \alpha_{Z F}(\mathbf{p}) \\
& =\underset{\mathbf{p} \in \mathrm{C}_{t}}{\arg \max } \sqrt{\frac{E_{T}}{\gamma_{Z F}(\mathbf{p})}} .
\end{aligned}
$$

From (3-17), the equivalence between the maximization of the detection 
signal-to-noise ratio and the minimization of $\gamma_{Z F}(\mathbf{p})$ is evident, given that the energy available at the transmitter, $E_{T}$, is fixed. Thus, the minimization of $\gamma_{Z F}(\mathbf{p})$ also corresponds to the minimization of the detection error probability. Finally, the antenna selection is indicated by the pattern $\mathbf{p}_{*}$ given by

$$
\mathbf{p}_{*}=\underset{\mathbf{p} \in \mathrm{C}_{t}}{\arg \min } \gamma_{Z F}(\mathbf{p})
$$

\subsection{3}

\section{Transmit Antenna Selection Approach for MMSE Precoding}

Now, we consider a system that uses the MMSE precoding technique, depicted in Section 2.4.2. Using (2-26), the precoding matrix for each subset $\mathbf{p}$ is given by

$$
\mathfrak{P}_{m}^{M M S E}(\mathbf{p})=\alpha_{M M S E}(\mathbf{p}) \mathfrak{D}_{m}^{H}(\mathbf{p}) \mathbf{F}_{m}(\mathbf{p}),
$$

where $\mathbf{F}_{m}$ is shown in (2-29), and with antenna selection, it is given by

$$
\mathbf{F}_{m}(\mathbf{p})=\left(\mathfrak{D}_{m}(\mathbf{p}) \mathfrak{D}_{m}^{H}(\mathbf{p})+\frac{\frac{1}{M} \sum_{m=1}^{M} \operatorname{Tr}\left(\mathfrak{R}_{\mathbf{n}_{m}}\right)}{E_{T}} \mathbf{I}_{K N_{R}}\right)^{-1} .
$$

The signal model in (2-11) can be expressed as

$$
\mathbf{r}_{m}=\mathfrak{D}_{m}(\mathbf{p}) \mathfrak{P}_{m}^{M M S E}(\mathbf{p}) \mathfrak{s}_{m}+\mathfrak{N}_{m}
$$

Unlike the ZF precoding, MMSE precoding does not remove the MUI completely. As previously shown in Section 2.1.2, the precoding matrix for the MU-MIMO-OFDM system at the $m$-th detection consists of a set of the precoding matrices of all users, that is $\mathfrak{P}_{m}=\left[\begin{array}{llll}\mathbf{P}_{m}^{(1)} & \mathbf{P}_{m}^{(2)} & \ldots & \mathbf{P}_{m}^{(K)}\end{array}\right]$. We know from (2-27) that minimizing the factor $\gamma_{M M S E}(\mathbf{p})$, we maximize $\alpha_{M M S E}(\mathbf{p})$ and therefore the desired energy at the receiver. However, it also maximizes the energy corresponding to the interference terms. Thus, minimization of the factor $\gamma_{M M S E}(\mathbf{p})$ does not guarantee an improvement in detection.

An approach to solve this issue, takes into account the interference terms. We define the matrices $\mathbf{W}_{m}(\mathbf{p})=\mathfrak{D}_{m}(\mathbf{p}) \tilde{\mathfrak{P}}_{m}^{M M S E}(\mathbf{p})$, where $\tilde{\mathfrak{P}}_{m}^{M M S E}$ (p) is the original precoding matrix without the factor $\alpha_{M M S E}(\mathbf{p})$, that is

$$
\tilde{\mathfrak{P}}_{m}^{M M S E}(\mathbf{p})=\mathfrak{D}_{m}^{H}(\mathbf{p}) \mathbf{F}_{m}(\mathbf{p})
$$

We also defined $\mathbf{W}_{m_{\text {diag }}}(\mathbf{p})$ which represent the diagonal matrix, containing the main diagonal elements of $\mathbf{W}_{m}(\mathbf{p})$ and $\overline{\mathbf{W}}_{m}(\mathbf{p})=\mathbf{W}_{m}(\mathbf{p})-\mathbf{W}_{m_{\text {diag }}}(\mathbf{p})$. 
Then the signal model in (3-21) can be rewritten as

$$
\begin{aligned}
\mathbf{r}_{m} & =\alpha_{M M S E}(\mathbf{p}) \mathbf{W}_{m}(\mathbf{p}) \mathfrak{s}_{m}+\mathfrak{N}_{m} \\
& =\alpha_{M M S E}(\mathbf{p}) \mathbf{W}_{m_{\text {diag }}}(\mathbf{p}) \mathfrak{s}_{m}+\alpha_{M M S E}(\mathbf{p}) \overline{\mathbf{W}}_{m}(\mathbf{p}) \mathfrak{s}_{m}+\mathfrak{N}_{m}
\end{aligned}
$$

where the first term contains the desired symbols and the second the multi-user interference. Now we define $\beta(\mathbf{p})$ as the total signal to total interference-plusnoise ratio, as

$$
\begin{aligned}
\beta(\mathbf{p}) & =\frac{\sum_{m=1}^{M} \operatorname{Tr}\left\{\alpha_{M M S E}^{2}(\mathbf{p}) \mathbf{W}_{m_{\text {diag }}}(\mathbf{p}) \boldsymbol{\Re}_{\mathbf{s}_{m}} \mathbf{W}_{m_{\text {diag }}}^{H}(\mathbf{p})\right\}}{\sum_{m=1}^{M}\left\{\operatorname{Tr}\left\{\alpha_{M M S E}^{2}(\mathbf{p}) \overline{\mathbf{W}}_{m}(\mathbf{p}) \boldsymbol{R}_{\mathbf{s}_{m}} \overline{\mathbf{W}}_{m}^{H}(\mathbf{p})\right\}+\operatorname{Tr}\left\{\mathfrak{R}_{\mathbf{n}_{m}}\right\}\right\}} \\
& =\frac{\sum_{m=1}^{M} \operatorname{Tr}\left\{\mathbf{W}_{m_{\text {diag }}}(\mathbf{p}) \mathbf{W}_{m_{\text {diag }}}^{H}(\mathbf{p})\right\}}{\sum_{m=1}^{M}\left\{\operatorname{Tr}\left\{\overline{\mathbf{W}}_{m}(\mathbf{p}) \overline{\mathbf{W}}_{m}^{H}(\mathbf{p})\right\}+\frac{\operatorname{Tr}\left\{\mathfrak{\Re}_{\mathbf{n}_{m}}\right\}}{E_{s} \alpha_{M M S E}^{2}(\mathbf{p})}\right\}}
\end{aligned}
$$

where the product $E_{s} \alpha_{M M S E}^{2}(\mathbf{p})$ can be deduced from (2-26), (2-28) and (2-29) and expressed as

$$
E_{s} \alpha_{M M S E}^{2}(\mathbf{p})=\frac{E_{T}}{\frac{1}{M} \sum_{m=1}^{M} \operatorname{Tr}\left(\mathbf{F}_{m}^{H}(\mathbf{p}) \mathfrak{D}_{m}(\mathbf{p}) \mathfrak{D}_{m}^{H}(\mathbf{p}) \mathbf{F}_{m}(\mathbf{p})\right)} .
$$

The strategy to find the best pattern $\mathbf{p}_{*}$ is through the maximization of $\beta(\mathbf{p})$. Then, the solution can be expressed as

$$
\mathbf{p}_{*}=\underset{\mathbf{p} \in \mathrm{C}_{t}}{\arg \max } \beta(\mathbf{p}) .
$$

\subsection{4}

\section{Simulation Results}

In this section, numerical results are presented to evaluate the BER performance in the MU-MIMO-OFDM scenario, using different approaches to select the best subset of antennas to transmit. The coefficients of the adopted discrete impulse response of the channels have the form shown in Section 3.1.4. The noise vector is a complex zero-mean Gaussian vector with circularly symmetric components and covariance matrix $\boldsymbol{R}_{\mathbf{n}_{m}}=\sigma_{n}^{2} \mathbf{I}_{K N_{R}}$. Results are expressed in terms of the signal-to-noise ratio, defined in Section 3.1.4. QPSK modulation is assumed and the length of the OFDM blocks is $M=64$.

\subsubsection{1}

\section{ZF Precoding}

When ZF precoding is performed by the BS, for a given channel realization and antenna pattern selection, the $k$-th user conditional BER is given 
by

$$
\operatorname{BER}_{k}\left(\gamma_{Z F}\right)=\mathrm{Q}\left(\sqrt{\frac{\alpha_{Z F}^{2}}{\sigma_{n}^{2}}}\right)=\mathrm{Q}\left(\sqrt{\frac{S N R}{\gamma_{Z F}}}\right),
$$

where $\mathrm{Q}($.$) is the Q-function defined as$

$$
\mathrm{Q}(x)=\frac{1}{\sqrt{2 \pi}} \int_{x}^{\infty} \exp \left(-\frac{u^{2}}{2}\right) d u,
$$

and the $k$-th user BER performance is

$$
\mathrm{BER}_{k}=\mathbb{E}\left[\mathrm{Q}\left(\sqrt{\frac{S N R}{\gamma_{Z F}}}\right)\right]
$$

In a semi-analytical approach we approximate (3-28) by

$$
\mathrm{BER}_{k} \cong \frac{1}{N_{C H}} \sum_{i=1}^{N_{C H}} \mathrm{Q}\left(\sqrt{\frac{S N R}{\gamma_{Z F_{i}}}}\right) .
$$

We note that (3-26) and the approximation (3-29), with $\gamma_{Z F}$ given by (2-25), are only applicable to the case of transmit antenna selection with ZF precoding.

Figure 3.8 compares the BER performance obtained with Monte Carlo simulation and with the semi-analytical approximation (3-29), when the ZF precoding scheme is employed. The semi-analytical approximation for the BER is in good agreement with the Monte Carlo simulated BER. These results were gathered after transmitting a total of $N_{S Y M}=76.8 \times 10^{6}$ symbols and $N_{D M}=20$ data-matrices for each of $N_{C H}=1 \times 10^{4}$ channel realizations. The main advantage of the use of the semi-analytical expression in (3-29) is less complexity by comparing with the simulation method.

Figure 3.9 shows the detection performance in ZF-precoded system, varying the number of available transmit antennas and the number of RF chains. These results evidence a significant improvement in terms of performance while keeping the number of RF chains fixed at the transmitter and adding the possibility of antenna selection. The combination $N_{T}=10, N_{R F}=6$ exhibits a gain of approximately $8 \mathrm{~dB}$ less energy than $N_{T}=6, N_{R F}=6$ (no antenna selection). On the other hand, curve $N_{T}=10, N_{R F}=8$ shows a trade-off between the number of available antennas to do antenna selection and an increase in the number of RF chains. The system with $N_{T}=10, N_{R F}=10$ depicts the best performance, but it also entails the highest cost and complexity. 


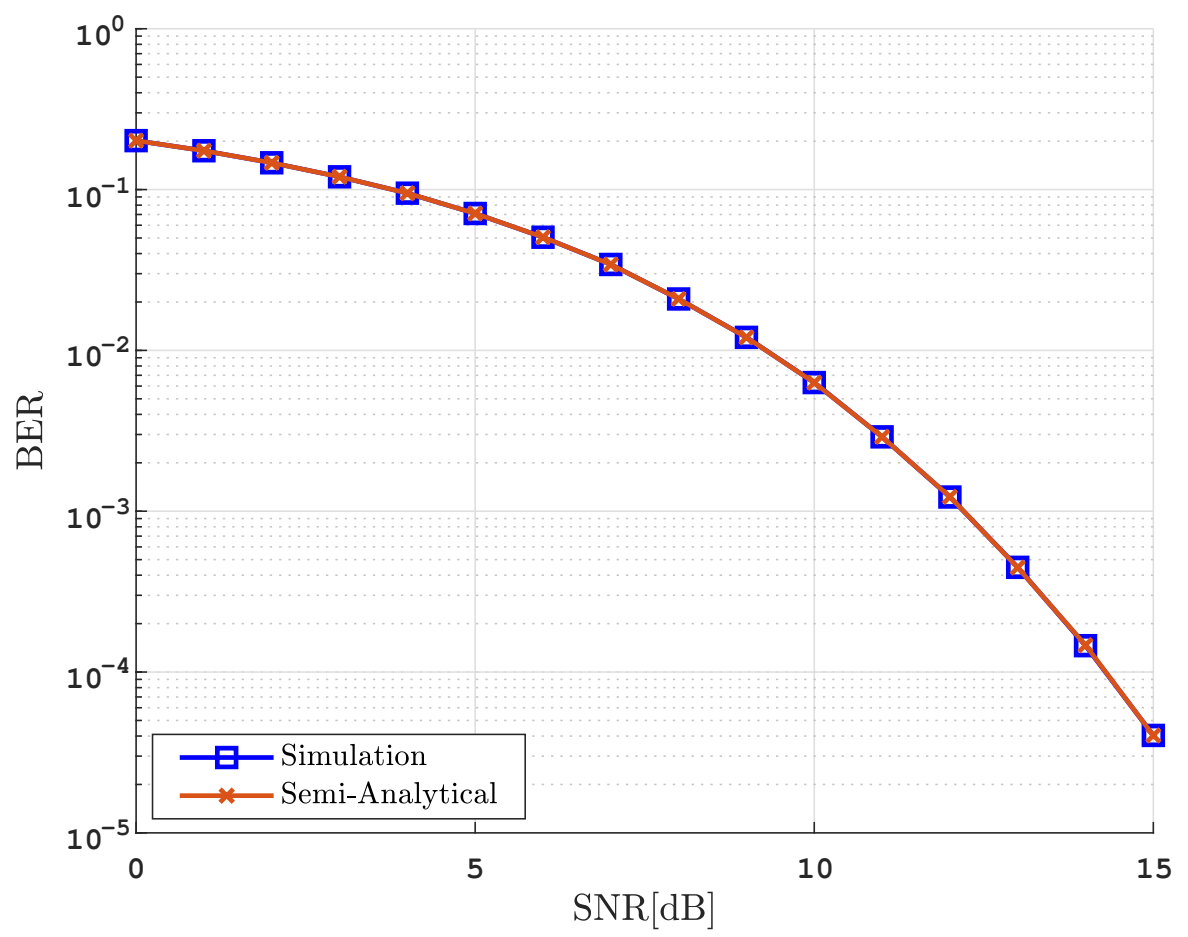

Figure 3.8: BER vs. SNR $[\mathrm{dB}]$ for $N_{T}=10, N_{R F}=10$, $N_{R}=3, K=2$ and $L=2$ in ZF-precoded system.

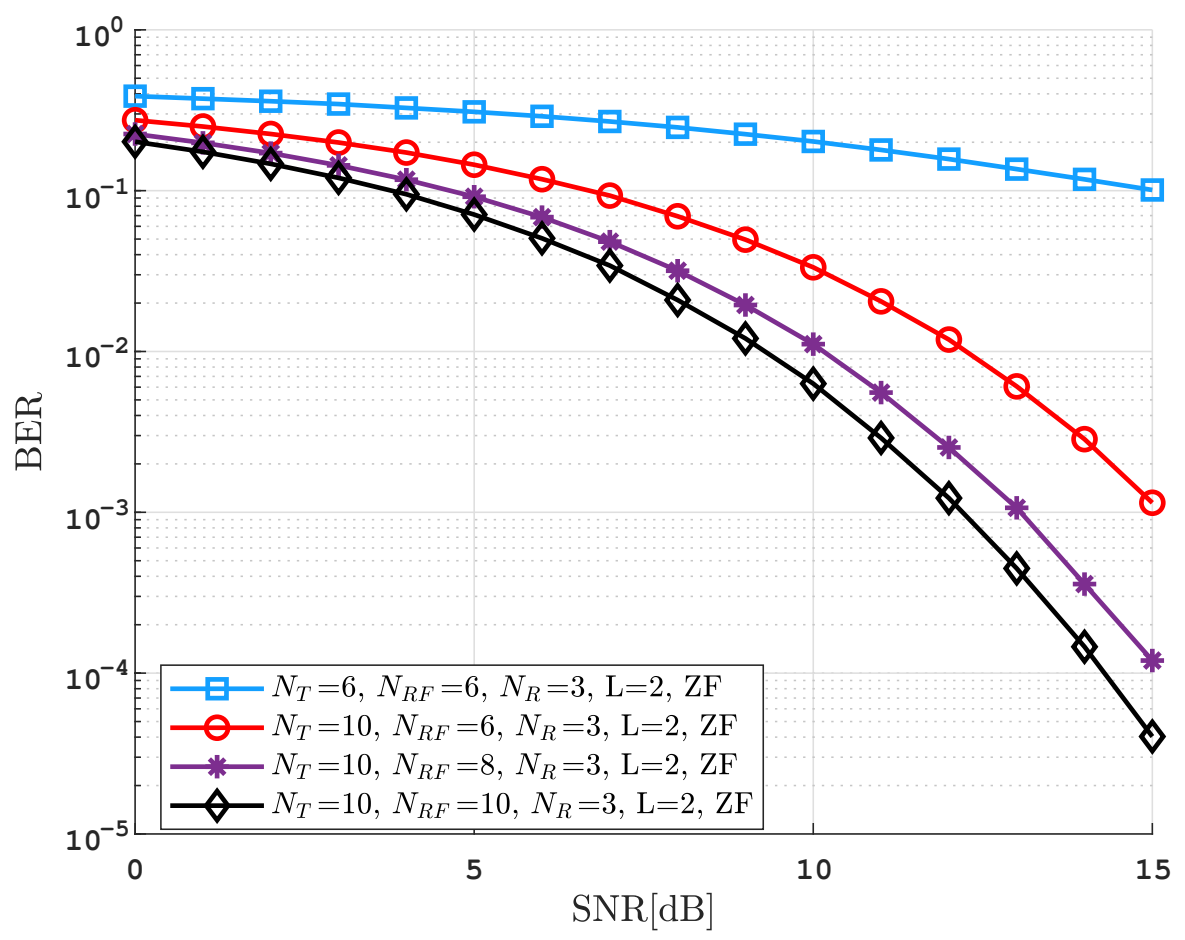

Figure 3.9: BER vs. SNR [dB] for transmit antenna selection using $\mathrm{ZF}$ precoding, $K=2$, and considering a different number of antennas and RF chains available at the transmitter. 


\subsubsection{2}

\section{MMSE Precoding}

Figure 3.10 shows the detection performance in MMSE-precoded system, for a different number of transmit antennas and RF chains. These results were gathered after transmitting a total of $N_{S Y M}=76.8 \times 10^{6}$ symbols and $N_{D M}=20$ data-matrices for each of $N_{C H}=1 \times 10^{4}$ channel realizations. As shown in the previous section, when transmitting antenna selection is employed and/or the number of RF chains grows, the system performance is improved.

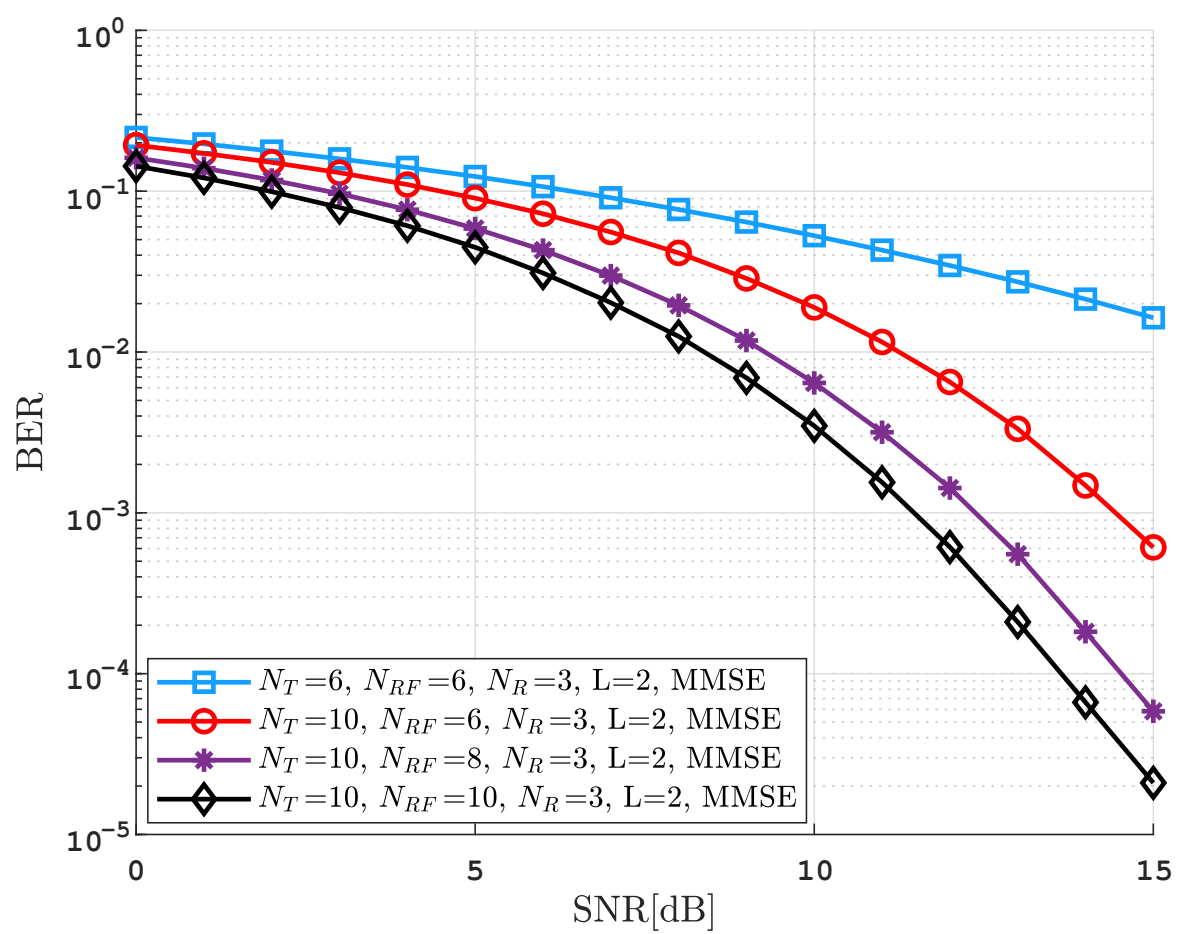

Figure 3.10: BER vs. SNR [dB] for transmit antenna selection using MMSE precoding, $K=2$, and considering a different number of antennas and RF chains available at the transmitter. 


\section{4}

\section{Receive Antennas Selection}

As presented in Chapter 3, the antenna selection at the transmitter improves the performance of the system without changes in terms of hardware complexity. In the same way, the antenna selection at the receiver can be employed as an excellent solution to achieve better values of BER.

Now, suppose that the $K$ users shown in Figure 4.1 are equipped with a reduced number, $N_{R R}$, of RF chains, and the number of available receiving antennas $N_{R}$ is higher than $N_{R R}$, i.e. $N_{R}>N_{R F}$. Note that the system has been generalized for MU-MIMO-OFDM scenario, and that the SU-MIMO-OFDM is a particular case by setting $K=1$.

The objective here is to select the best subset of $N_{R R}$ receive antennas according to the current characteristics of the transmission channels.

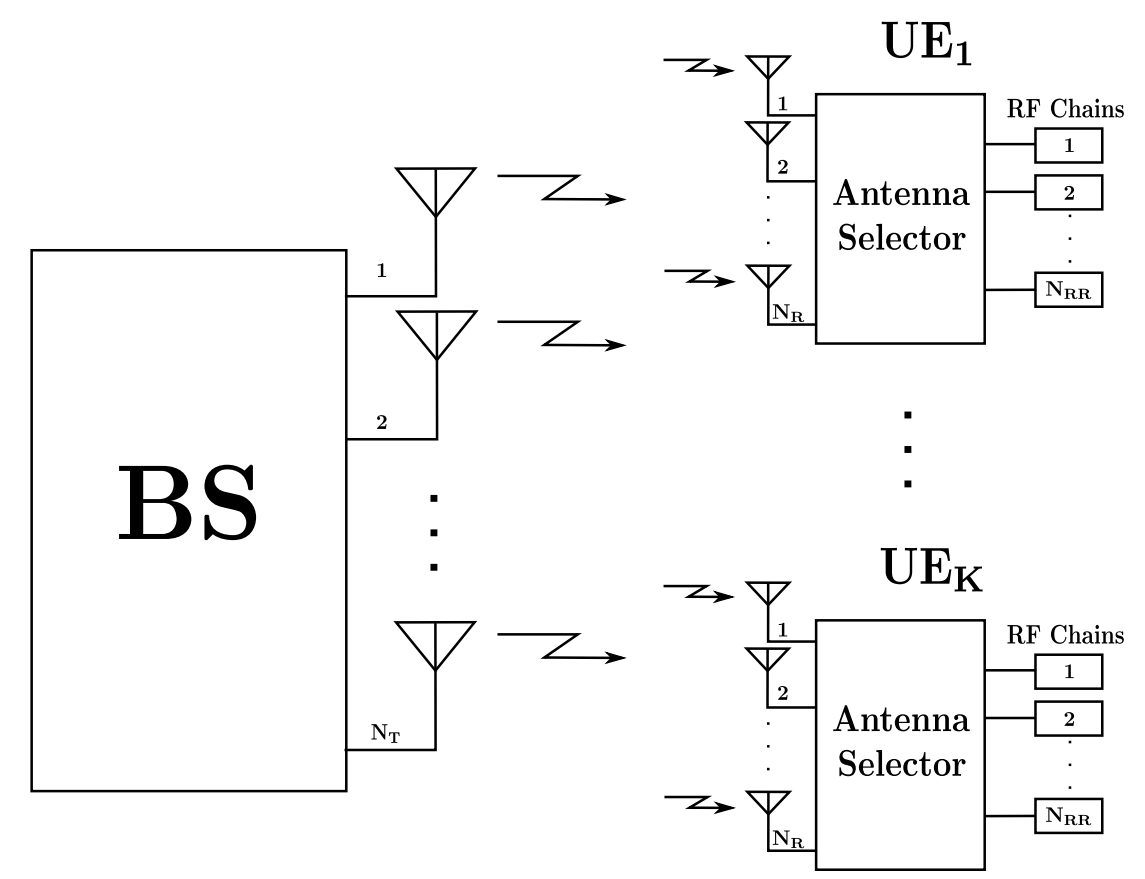

Figure 4.1: MIMO-OFDM system with antenna selection at the receiver.

In this chapter, we present a general model to describe the receive antennas selection for SU-MIMO-OFDM (non-precoded) and MU-MIMOOFDM (precoded) systems. Receive antenna selection strategies are proposed for all cases. Simulation results are presented, employing ZF and MMSE 
equalizers for the SU-MIMO-OFDM case, as well as ZF and MMSE precoders in the MU-MIMO-OFDM scenario.

\section{1}

\section{SU-MIMO-OFDM system (non-precoded)}

\subsection{1}

\section{Receive Antenna Selection Model for SU-MIMO-OFDM systems}

Analogously to the vector $\mathbf{p}$ that was presented in Chapter 3, we define a vector $\mathbf{q} \in\{1,0\}^{N_{R}}$ that indicates the subset of antennas that are activated, from the entire set of $N_{R}$ antennas. A one value at any position in vector $\mathbf{q}$ indicates that the corresponding antenna is activated, whereas a zero value indicates that the system does not use this antenna for reception.

The corresponding effective channel matrix, $\mathbb{D}_{m}(\mathbf{q}) \in \mathbb{C}^{N_{R R} \times N_{T}}$ is obtained by selecting the columns indexed by q. Thus,

$$
\tilde{\mathbb{D}}_{m}(\mathbf{q})=\tilde{\mathbf{U}}(\mathbf{q}) \mathbb{D}_{m}
$$

where $\tilde{\mathbf{U}}(\mathbf{q}) \in \mathbb{C}^{N_{R R} \times N_{R}}$ is the matrix obtained from $\mathbf{I}_{N_{R}}$ eliminating its $i$-th row if the $i$-th component of vector $\mathbf{q}$ is zero. It then results that $\tilde{\mathbf{U}}^{T}(\mathbf{q}) \tilde{\mathbf{U}}(\mathbf{q})=\operatorname{diag}(\mathbf{q})=\tilde{\mathbf{B}}(\mathbf{q})$ and $\tilde{\mathbf{U}}(\mathbf{q}) \tilde{\mathbf{U}}^{T}(\mathbf{q})=\mathbf{I}_{N_{R R}}$.

Let $C_{r}$ be the set of possible $\mathbf{q}$ patterns indicating $N_{R R^{-}}$selected out of $N_{R}$ antennas, then the total number of possible combinations is given by

$$
\left|C_{r}\right|=\left(\begin{array}{c}
N_{R} \\
N_{R R}
\end{array}\right)=\frac{N_{R} !}{\left(N_{R}-N_{R R}\right) ! N_{R R} !} .
$$

The BS selects the most appropriate set of antennas that should be activated during a OFDM symbol period and then it must notify to the receiver which of the $C_{r}$ possible patterns is chosen, in order to improve the performance of the system.

\subsection{2}

\section{Receive Antenna Selection using MIA}

As presented in Section 3.1.2, mutual information can be used as a metric to select the best subset of antennas to transmit. Similarly, MIA can be used to choose antennas at the receiver. The BS calculates the mutual information for all patterns of the vector $\mathbf{q}$, and informs to the receiver which one results in the highest value. The selection is made taking into account the effective channel matrix $\mathbb{D}_{m}(\mathbf{q})$ for each of the $M$ detections. Thus, the mutual information can be calculated as 


$$
\mathbf{I}(\mathbf{q})=\sum_{m=1}^{M} \log _{2} \operatorname{det}\left[\frac{E_{s}}{\sigma_{n}^{2}} \tilde{\mathbb{D}}_{m}(\mathbf{q}) \tilde{\mathbb{D}}_{m}^{H}(\mathbf{q})+\mathbf{I}_{N_{R R}}\right] .
$$

Hence, the antenna selection is indicated by the pattern $\mathbf{q}_{*}$ given by

$$
\mathbf{q}_{*}=\underset{\mathbf{q} \in \mathrm{C}_{r}}{\arg \max } \mathbf{I}(\mathbf{q}) .
$$

\subsection{3}

\section{Receive Antenna Selection minimizing the MSE}

MSE approach can also be used to select antennas at the receiver in SUMIMO-OFDM scenario. This method, as also shown in the previous chapter, is a more specific solution by comparing it with MIA, because it depends on the current equalizer.

\subsubsection{1}

\section{MSE Minimizing in ZF-equalized receiver}

Taking into account the expression obtained from (3-6), we can express the MSE in a ZF-equalized receiver as

$$
M S E_{Z F}=\sigma_{n}^{2} \sum_{m=1}^{M} \operatorname{Tr}\left\{\left(\mathbb{D}_{m}^{H} \mathbb{D}_{m}\right)^{-1}\right\} .
$$

Therefore, the antenna selection, indicated by the pattern $\mathbf{q}_{*}$, can be calculated as

$$
\mathbf{q}_{*}=\underset{\mathbf{q}}{\arg \min } \sigma_{n}^{2} \sum_{m=1}^{M} \operatorname{Tr}\left\{\left(\tilde{\mathbb{D}}_{m}^{H}(\mathbf{q}) \tilde{\mathbb{D}}_{m}(\mathbf{q})\right)^{-1}\right\}
$$

\subsubsection{2}

\section{MSE Minimizing in MMSE-equalized receiver}

MMSE equalizer takes into account the effect of the noise, and the price to pay is the non-removed channel matrix after equalization. In this case, the MSE can be calculated from (3-8) as

$$
M S E_{M M S E}=\sum_{m=1}^{M} \mathbb{E}\left\{\left\|\hat{\mathbf{x}}_{m}^{M M S E}-\mathbf{x}_{m}\right\|^{2}\right\} .
$$


Using (2-18) and (2-19), we can show that the solution for (4-7) can be written as

$$
M S E_{M M S E}=\sigma_{n}^{2} \sum_{m=1}^{M} \operatorname{Tr}\left\{\left(\mathbb{D}_{m}^{H} \mathbb{D}_{m}+\frac{\sigma_{n}^{2}}{E_{s}} \mathbf{I}_{N_{T}}\right)^{-1}\right\}
$$

and the best pattern for the vector $\mathbf{q}$ can be calculated by minimizing $M S E_{M M S E}$ and is given by

$$
\mathbf{q}_{*}=\underset{\mathbf{q}}{\arg \min } \sigma_{n}^{2} \sum_{m=1}^{M} \operatorname{Tr}\left\{\left(\tilde{\mathbb{D}}_{m}^{H}(\mathbf{q}) \tilde{\mathbb{D}}_{m}(\mathbf{q})+\frac{\sigma_{n}^{2}}{E_{s}} \mathbf{I}_{N_{T}}\right)^{-1}\right\} .
$$

\subsection{4}

\section{Simulation Results}

In this section, numerical results are presented to evaluate the BER performance in the SU-MIMO-OFDM scenario, using different approaches to select the best subset of antennas to be activated for reception.

The coefficients of the adopted discrete impulse response of the channels have the form illustrated in Section 3.1.4. The noise vector $\tilde{\mathbf{n}}_{m}$ in (2-5) is a complex zero-mean Gaussian vector with circularly symmetric components and covariance matrix $\mathbf{K}_{\tilde{\mathbf{n}}}=\sigma_{n}^{2} \mathbf{I}_{N_{R}}$. Results are expressed in terms of the signalto-noise ratio, defined in Section 3.1.4 and QPSK modulation is assumed. The length of the OFDM blocks is $M=64$.

\subsubsection{1}

\section{ZF and MMSE for MIA}

Figures 4.2 and 4.3 depict the detection performance for a range of SNR, considering ZF and MMSE equalization, respectively, and varying the number of available receive antennas, while the number of receive $R F$ chains and transmit antennas is kept fixed. In both cases, when MIA is employed as the antenna selection strategy, the performance of the system is improved. Furthermore, the improvement is greater as the number of antennas available at the receiver increases. The gain for systems with $N_{R}=10$, in Figures 4.2 and 4.3 is approximately $9.8 \mathrm{~dB}$ and $9.5 \mathrm{~dB}$, respectively, compared to the case where $N_{R}=2$. These results were obtained after transmitting a total of $N_{S Y M}=12.8 \times 10^{7}$ symbols and $N_{D M}=20$ data-matrices for each of $N_{C H}=5 \times 10^{4}$ channel realizations. 


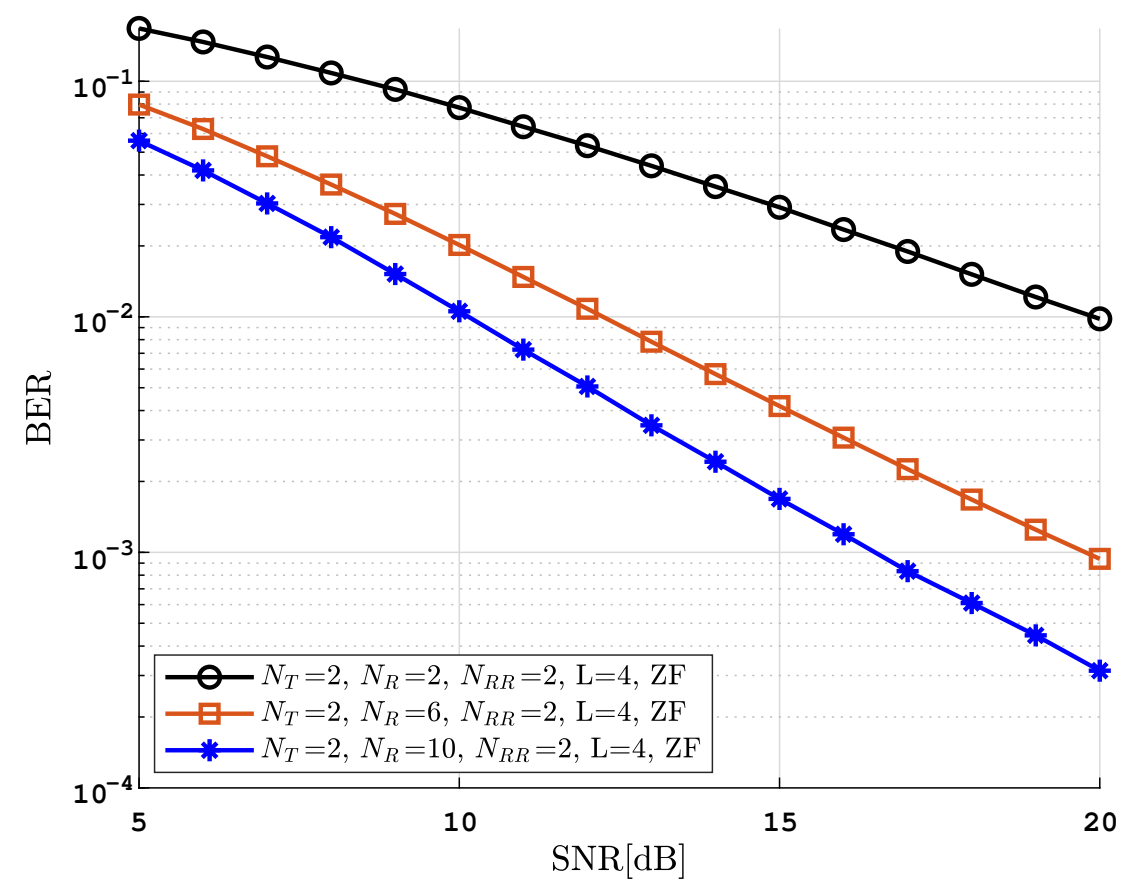

Figure 4.2: BER vs. SNR [dB] for receive antenna selection using Mutual Information Approach, ZF equalizer, and considering a different number of available antennas at the receiver.

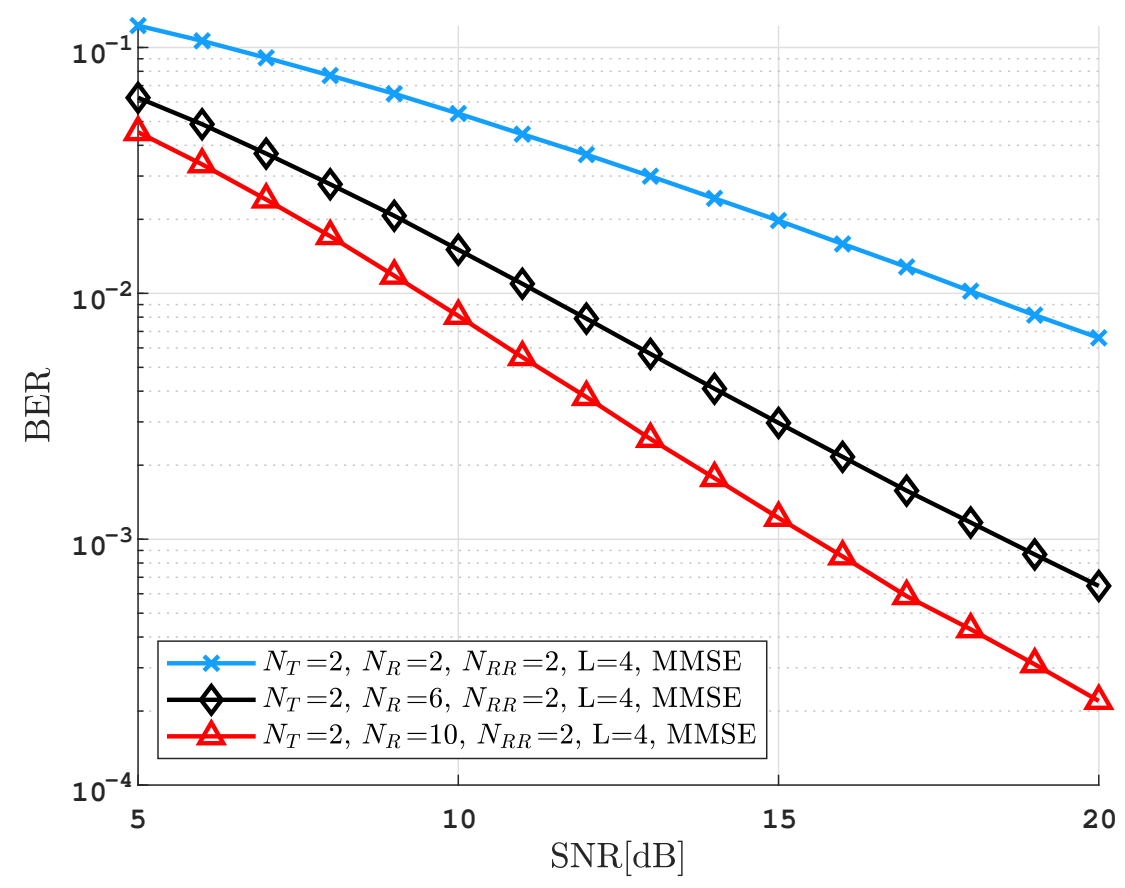

Figure 4.3: BER vs. SNR [dB] for receive antenna selection using Mutual Information Approach, MMSE equalizer, and considering a different number of available antennas at the receiver. 


\subsubsection{2}

\section{ZF and MMSE for MSE approach}

Simulation in Figures 4.4 and 4.5 show the detection performance for ZF and MMSE equalization, respectively. They both were obtained using the MSE approach as the antenna selection strategy. We can see that the system with $N_{R}=10$, in both cases, requires significantly less energy (greater than $10 \mathrm{~dB}$ in number) compared to the case where $N_{R}=2$, when no antenna selection strategies are employed. These results were gathered after transmitting a total of $N_{S Y M}=12.8 \times 10^{7}$ symbols and $N_{D M}=20$ data-matrices for each of $N_{C H}=5 \times 10^{4}$ channel realizations.

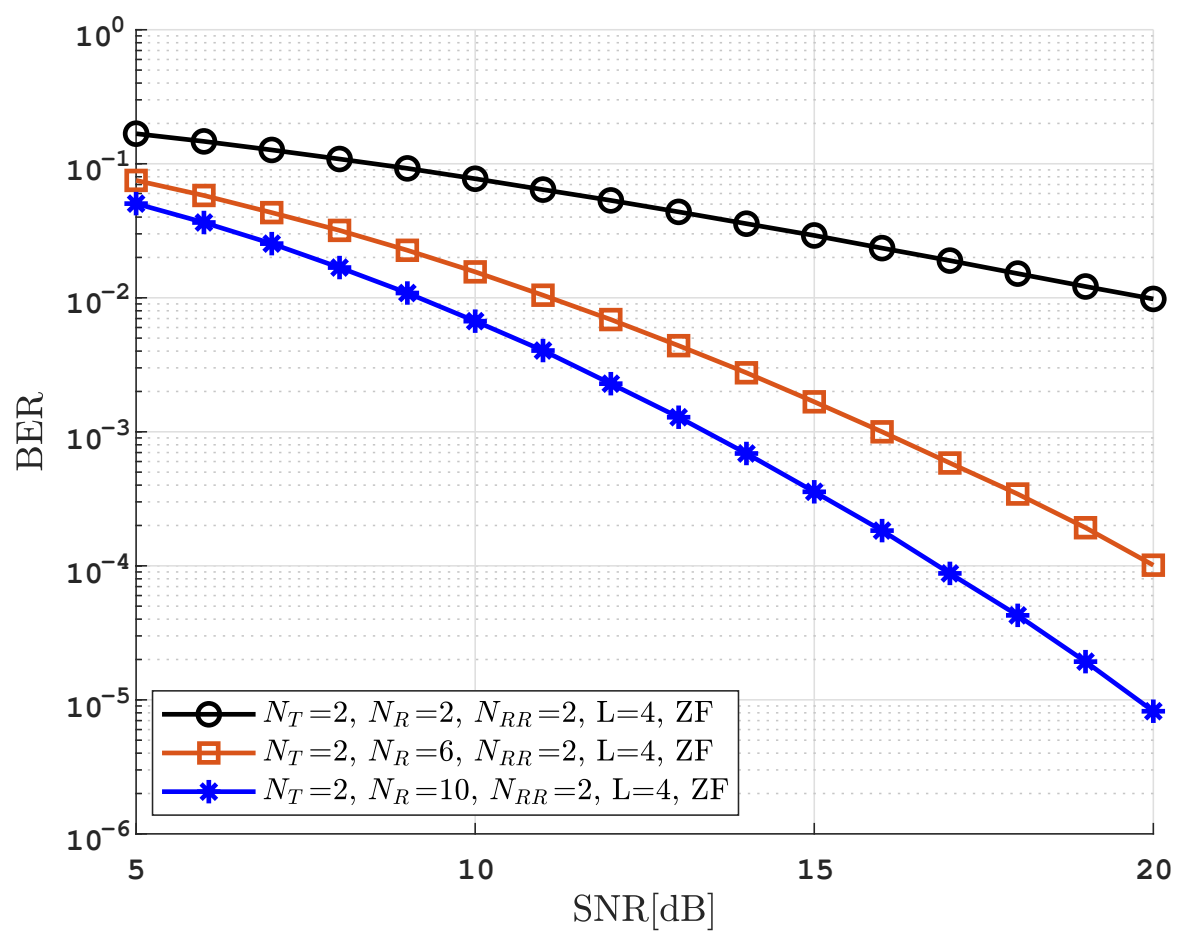

Figure 4.4: BER vs. SNR $[\mathrm{dB}]$ for receive antenna selection using MSE Approach, ZF equalizer and considering different number of available antennas at the receiver. 


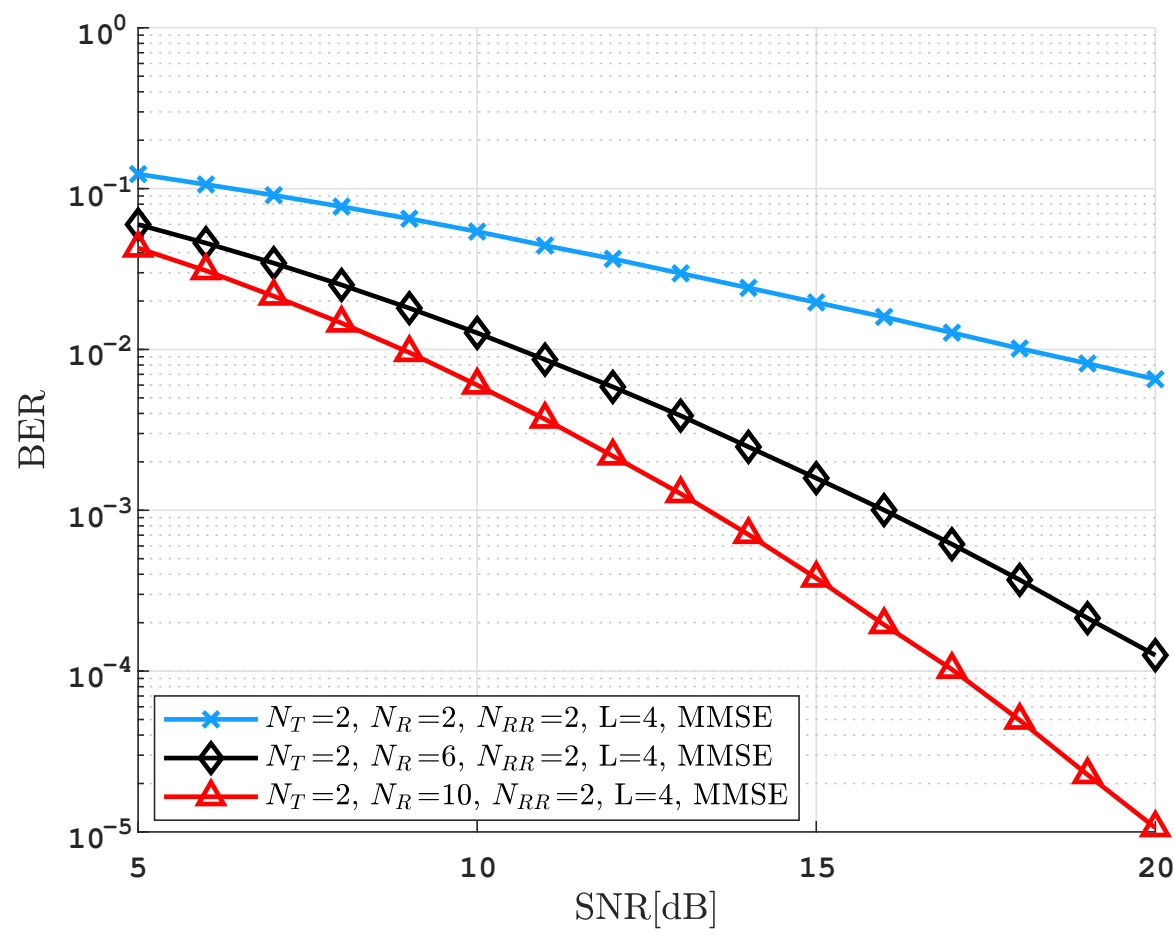

Figure 4.5: BER vs. SNR [dB] for receive antenna selection using MSE Approach, MMSE equalizer and considering different number of available antennas at the receiver.

\subsubsection{3 \\ Comparisons}

In Figures 4.6 and 4.7, the improvement of the system using the antenna selection schemes is noticeable. The result for random selection is barely acceptable and similar to the performance when no antenna selection method is employed, i.e., curves $N_{R}=2$.

As shown in Section 3.1.4.3, the performance at higher SNR values is better when the system using MSE rather than MIA. When the system chooses the best antenna pattern using MSE, the system computes the minimum mean square error, based on the current equalizer. In contrast, MIA achieves a poorer detection performance, but its complexity is significantly lower compared to the use of MSE. 


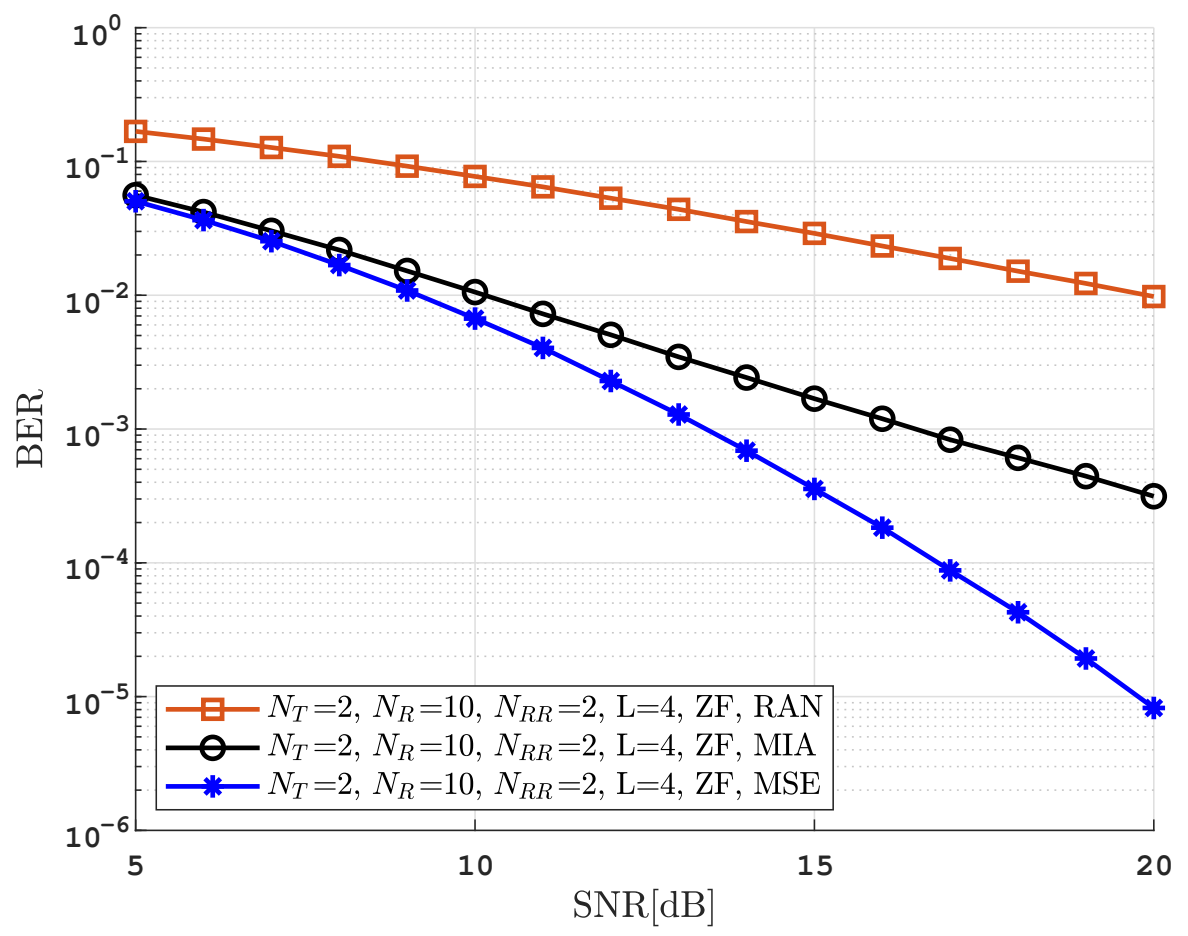

Figure 4.6: BER vs. SNR [dB] for receive antenna selection using the proposed methods (MIA and MSE) and random selection. Results were obtained employing ZF equalizer.

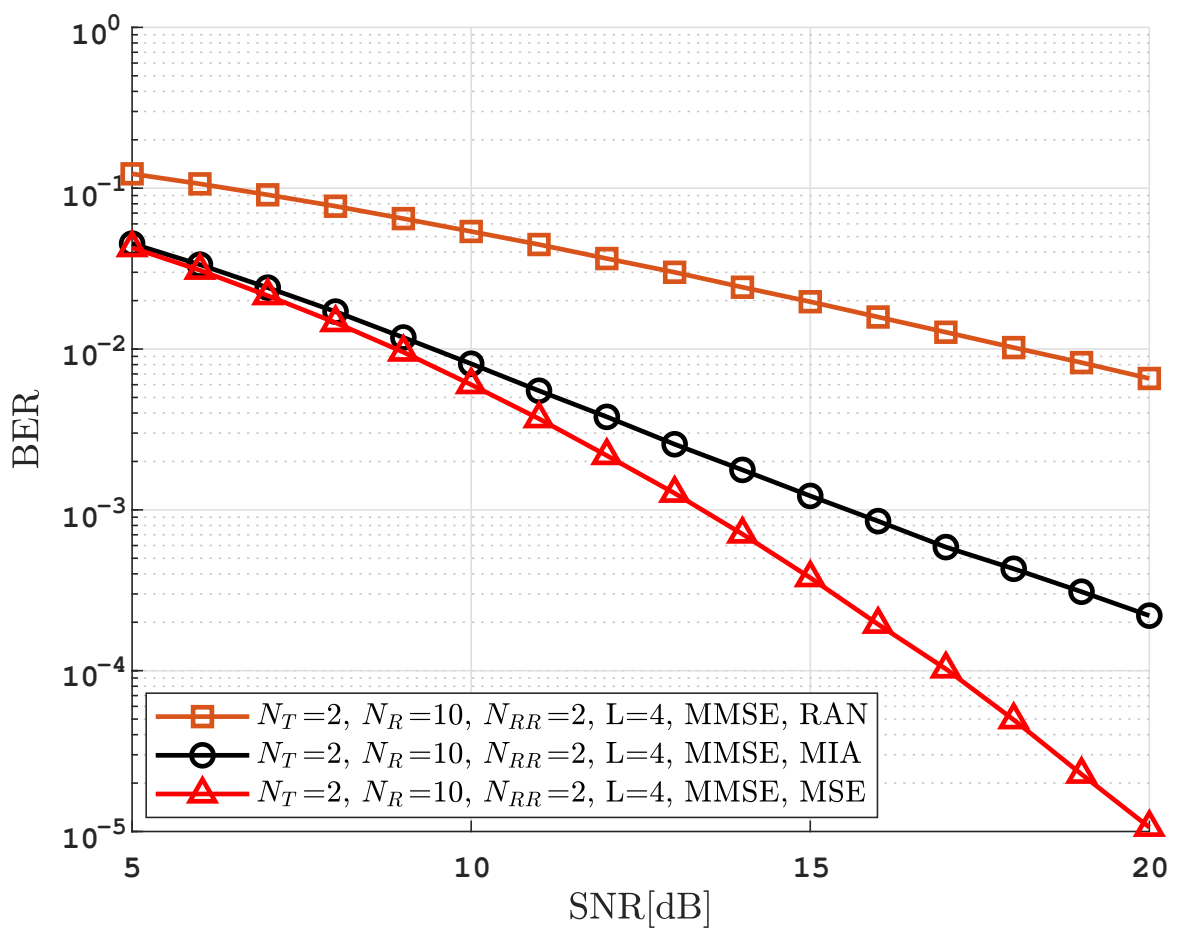

Figure 4.7: BER vs. SNR [dB] for receive antenna selection using the proposed methods (MIA and MSE) and random selection. Results were obtained employing MMSE equalizer. 


\section{2}

\section{MU-MIMO-OFDM system (precoded)}

\subsection{1}

\section{Receive Antenna Selection Model for MU-MIMO-OFDM systems}

To follow the procedure used to select antennas in previous sections, we define a vector $\tilde{\mathbf{q}}=\left[\mathbf{q}_{1}^{T}, \mathbf{q}_{2}^{T}, \ldots, \mathbf{q}_{K}^{T}\right]^{T}$, which contains the information on the state of the antennas (activated or deactivated). Each entry $\mathbf{q}_{k}$, $k=1,2, \ldots, K$, is similar to the vector $\mathbf{q}$ defined in Section 4.1.1, and refers to the pattern used by each user. The corresponding effective channel matrix $\mathfrak{D}^{\prime}{ }_{m}(\tilde{\mathbf{q}}) \in \mathbb{C}^{K N_{R R} \times N_{T}}$, following the system model proposed in (2-11), is obtained by selecting the columns indexed by $\tilde{\mathbf{q}}$. Thus,

$$
\mathfrak{D}^{\prime}{ }_{m}(\tilde{\mathbf{q}})=\mathbf{U}^{\prime}(\tilde{\mathbf{q}}) \mathfrak{D}_{m},
$$

where $\mathbf{U}^{\prime}(\tilde{\mathbf{q}}) \in \mathbb{C}^{K N_{R R} \times K N_{R}}$ is obtained from $\mathbf{I}_{K N_{R}}$, suppressing its $i$ th row, when the $i$ th component of vector $\tilde{\mathbf{q}}$ is zero and can be expressed as

$$
\mathbf{U}^{\prime}(\tilde{\mathbf{q}})=\left[\begin{array}{cccc}
\tilde{\mathbf{U}}\left(\mathbf{q}_{1}\right) & 0 & \cdots & 0 \\
0 & \tilde{\mathbf{U}}\left(\mathbf{q}_{2}\right) & \cdots & 0 \\
\vdots & \vdots & \ddots & \vdots \\
0 & 0 & 0 & \tilde{\mathbf{U}}\left(\mathbf{q}_{K}\right)
\end{array}\right]
$$

\subsection{2}

\section{Receive Antenna Selection Approach for ZF Precoding}

When the BS employs the ZF precoding to process the information symbols vectors that should be transmitted to each user, the precoding matrix can be deduced from (2-23) and (4-10), and is given by

$$
\mathfrak{P}_{m}^{Z F}(\tilde{\mathbf{q}})=\alpha_{Z F}(\tilde{\mathbf{q}}) \mathfrak{D}_{m}^{\prime H}(\tilde{\mathbf{q}})\left(\mathfrak{D}_{m}^{\prime}(\tilde{\mathbf{q}}) \mathfrak{D}_{m}^{\prime H}(\tilde{\mathbf{q}})\right)^{-1}
$$

Using (4-11), the received vector for the ZF-precoded system can be written as

$$
\mathbf{r}_{m}=\alpha_{Z F}(\tilde{\mathbf{q}}) \mathfrak{s}_{m}+\mathfrak{N}_{m}
$$

Finally, the antenna selection is indicated by the pattern $\tilde{\mathbf{q}}_{*}$ given by

$$
\begin{aligned}
\tilde{\mathbf{q}}_{*} & =\underset{\tilde{\mathbf{q}} \in \mathrm{C}_{r}}{\arg \max } \alpha_{Z F}(\tilde{\mathbf{q}}) \\
& =\underset{\tilde{\mathbf{q}} \in C_{r}}{\arg \max } \sqrt{\frac{E_{T}}{\gamma_{Z F}(\tilde{\mathbf{q}})}} \\
& =\underset{\tilde{\mathbf{q}} \in \mathrm{C}_{r}}{\arg \min } \gamma_{Z F}(\tilde{\mathbf{q}}) .
\end{aligned}
$$


where $\gamma_{Z F}$ is obtained from $(2-25)$.

\subsection{3}

\section{Receive Antenna Selection Approach for MMSE Precoding}

Considering the MMSE precoding technique, addressed in Section 2.4.2, the expression of the precoding matrix for a given pattern $\tilde{\mathbf{q}}$ is given by

$$
\mathfrak{P}_{m}^{M M S E}(\tilde{\mathbf{q}})=\alpha_{M M S E}(\tilde{\mathbf{q}}) \mathfrak{D}_{m}^{\prime H}(\tilde{\mathbf{q}}) \mathbf{F}_{m}(\tilde{\mathbf{q}}),
$$

where $\mathbf{F}_{m}$ is shown in (2-29). Then, the signal model in (2-11) can be expressed as

$$
\mathbf{r}_{m}=\mathfrak{D}_{m}^{\prime}(\tilde{\mathbf{q}}) \mathfrak{P}_{m}^{M M S E}(\tilde{\mathbf{q}}) \mathfrak{s}_{m}+\mathfrak{N}_{m}
$$

Following the procedure to define a metric for the MMSE-precoded system, we can use the matrices $\mathbf{W}_{m}, \mathbf{W}_{m_{\text {diag }}}$ and $\overline{\mathbf{W}}_{m}$, defined in Section 3.2 .3 , and therefore, the received vector can be expressed as

$$
\begin{aligned}
\mathbf{r}_{m} & =\alpha_{M M S E}(\tilde{\mathbf{q}}) \mathbf{W}_{m}(\tilde{\mathbf{q}}) \mathfrak{s}_{m}+\mathfrak{N}_{m} \\
& =\alpha_{M M S E}(\tilde{\mathbf{q}}) \mathbf{W}_{m_{\text {diag }}}(\tilde{\mathbf{q}}) \mathfrak{s}_{m}+\alpha_{M M S E}(\tilde{\mathbf{q}}) \overline{\mathbf{W}}_{m}(\tilde{\mathbf{q}}) \mathfrak{s}_{m}+\mathfrak{N}_{m}
\end{aligned}
$$

Now, $\beta(\tilde{\mathbf{q}})$ is the total signal to total interference-plus-noise ratio, and can be obtained from

$$
\begin{aligned}
\beta(\tilde{\mathbf{q}}) & =\frac{\sum_{m=1}^{M} \operatorname{Tr}\left\{\alpha_{M M S E}^{2}(\tilde{\mathbf{q}}) \mathbf{W}_{m_{\text {diag }}}(\tilde{\mathbf{q}}) \mathfrak{\Re}_{\mathbf{s}_{m}} \mathbf{W}_{m_{\text {diag }}}^{H}(\tilde{\mathbf{q}})\right\}}{\sum_{m=1}^{M}\left\{\operatorname{Tr}\left\{\alpha_{M M S E}^{2}(\tilde{\mathbf{q}}) \overline{\mathbf{W}}_{m}(\tilde{\mathbf{q}}) \mathfrak{\Re}_{\mathbf{s}_{m}} \overline{\mathbf{W}}_{m}^{H}(\tilde{\mathbf{q}})\right\}+\operatorname{Tr}\left\{\mathfrak{\Re}_{\mathbf{n}_{m}}\right\}\right\}} \\
& =\frac{\sum_{m=1}^{M} \operatorname{Tr}\left\{\mathbf{W}_{m_{\text {diag }}}(\tilde{\mathbf{q}}) \mathbf{W}_{m_{\text {diag }}}^{H}(\tilde{\mathbf{q}})\right\}}{\sum_{m=1}^{M}\left\{\operatorname{Tr}\left\{\overline{\mathbf{W}}_{m}(\tilde{\mathbf{q}}) \overline{\mathbf{W}}_{m}^{H}(\tilde{\mathbf{q}})\right\}+\frac{\operatorname{Tr}\left\{\mathfrak{R}_{\mathbf{n}_{m}}\right\}}{E_{s} \alpha_{M M S E}^{2}(\tilde{\mathbf{q}})}\right\}}
\end{aligned}
$$

where the product $E_{s} \alpha_{M M S E}^{2}(\tilde{\mathbf{q}})$ can be deduced from (2-26), (2-28) and (2-29) and expressed as

$$
E_{s} \alpha_{M M S E}^{2}(\tilde{\mathbf{q}})=\frac{E_{T}}{\frac{1}{M} \sum_{m=1}^{M} \operatorname{Tr}\left(\mathbf{F}_{m}^{H}(\tilde{\mathbf{q}}) \mathfrak{D}^{\prime}{ }_{m}(\tilde{\mathbf{q}}) \mathfrak{D}_{m}^{\prime H}(\tilde{\mathbf{q}}) \mathbf{F}_{m}(\tilde{\mathbf{q}})\right)}
$$

The strategy to find the best pattern $\tilde{\mathbf{q}}_{*}$ is through the maximization of $\beta(\tilde{\mathbf{q}})$. Then, the solution can be expressed as

$$
\tilde{\mathbf{q}}_{*}=\underset{\tilde{\mathbf{q}} \in \mathrm{C}_{r}}{\arg \max } \beta(\tilde{\mathbf{q}}) .
$$




\subsection{4}

\section{Simulation Results}

In this section, numerical results are presented to evaluate the BER performance in the MU-MIMO-OFDM scenario, using different approaches to select the best subset of antennas to transmit. The results were gathered after transmitting a total of $N_{S Y M}=76.8 \times 10^{6}$ symbols and $N_{D M}=20$ datamatrices for each of $N_{C H}=1 \times 10^{4}$ channel realizations. The coefficients of the adopted discrete impulse response of the channels have the form shown in Section 3.1.4. The noise vector is a complex zero-mean Gaussian vector with circularly symmetric components and covariance matrix $\mathfrak{R}_{\mathbf{n}_{m}}=\sigma_{n}^{2} \mathbf{I}_{K N_{R R}}$. Results are expressed in terms of the signal-to-noise ratio, defined in Section 3.1.4. QPSK modulation is assumed and the length of the OFDM blocks is $M=64$.

\subsubsection{1}

\section{ZF Precoding}

Figure 4.8 shows the detection performance in ZF-precoded system, using the proposed method and random selection. The curve $N_{T}=10, N_{R}=4$ and $N_{R R}=2$ (the proposed method) achieves the best performance, i.e., $2 \mathrm{~dB}$ less energy than the others. Random selection (RAN) shows an equal performance by comparing with $N_{T}=10, N_{R}=2$ and $N_{R R}=2$ (no antenna selection).

This simulation was feasible because the number of possible combinations to test during the selection process was small. It can be shown that the number of combinations using exhaustive search in MU receive antenna selection scenario is given by $\left|C_{r}\right|^{K}$, where $\left|C_{r}\right|$ can be calculated from (4-2). In order to minimize the simulation complexity, researchers have proposed many suboptimum algorithms, and they gathered results near to the optimal one. For instance, [11] proposes a sub-optimum method to simplify the receive antenna selection process in ZF-precoded systems. 


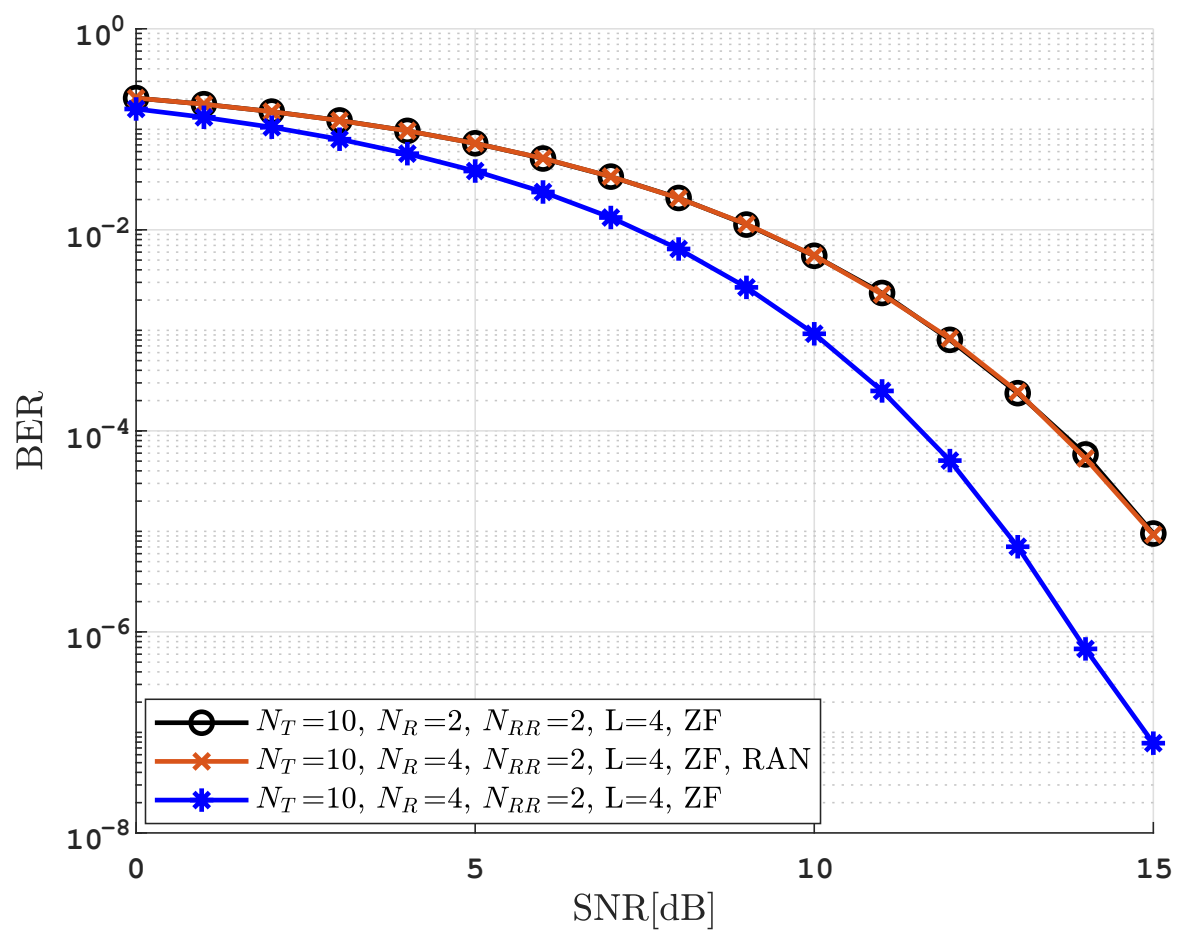

Figure 4.8: BER vs. SNR $[\mathrm{dB}]$ for receive antenna selection using $\mathrm{ZF}$ precoding, $K=3$, and considering a different number of available antennas at the receiver.

\subsubsection{2}

\section{MMSE Precoding}

Figure 4.9 shows the detection performance in MMSE-precoded system for a different number of receiving antennas. This figure depicts the improvement when receiving antenna selection is employed. 


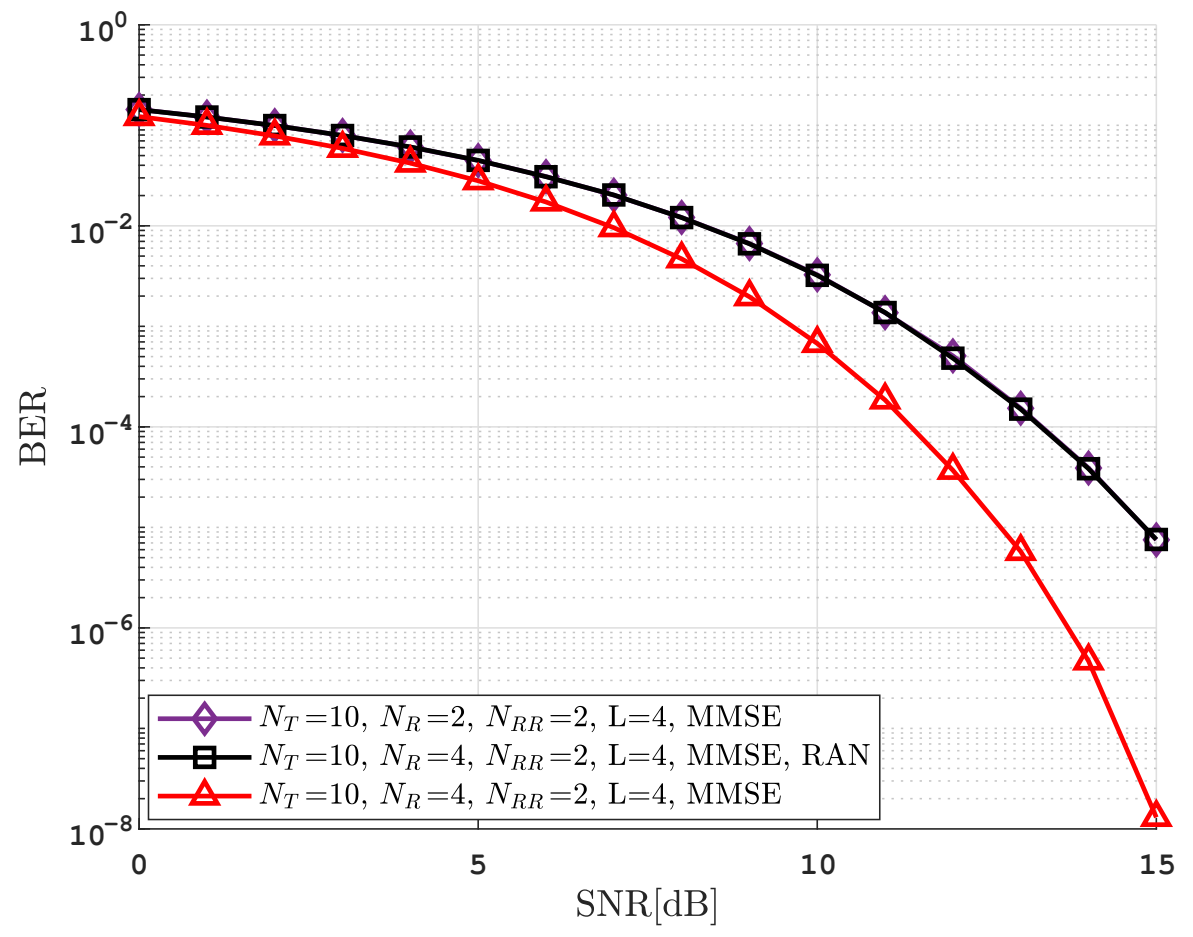

Figure 4.9: BER vs. SNR [dB] for receive antenna selection using MMSE precoding, $K=3$, and considering a different number of available antennas at the receiver. 


\section{5}

\section{Conclusions and Future Work}

This dissertation focused on the antenna selection problem in MIMOOFDM systems. Some strategies were proposed to select antennas in both scenarios, SU and MU. The guideline behind the antenna selection approaches was based on the reduced number of RF chains, and from there, improve the system performance by increasing the number of antennas available to select.

In Chapter 2, we have presented a general description of the principles associated with the design and implementation of MIMO-OFDM systems. A detailed signal modelling for $\mathrm{SU}$ and $\mathrm{MU}$ scenario is presented. In the model herein, a clear approach that converts the detection of parallel OFDM blocks emitted by the transmit antennas into independent flat-fading MIMO detection decoupled per OFDM subsymbol is developed. We have also outlined a review of the mobile radio channel characterization, highlighting the most common effects on the RF signal when it passes from the transmitter to the receiver. Linear equalizers, such as ZF and MMSE, have been presented, as well as the precoding techniques employed in this work.

In Chapter 3, we have developed strategies to implement transmit antenna selection, relying on the maximization of the mutual information or the minimization of the mean square error in SU scenario. In the MU case, we have proposed methods based on the maximization of the SNR for the ZF-precoded case and based on the maximization of the SINR for the MMSEprecoded case.

The approaches presented for transmitting antenna selection were extended for receiving antenna selection in Chapter 4. Here, the system model was different and more complex in MU scenario, because it takes into account the possible combinations for each user. The selection is performed by the BS and it relies on the search of the best antenna pattern for each user based on a given metric. Then, the BS informs each user which antenna pattern should be used.

Simulation results, showing the performance of the proposed selection approaches have been presented. Numerical results evidenced that the availability of extra antennas at the transmitter or at the receiver results in significant system performance improvements and that the proposed strategies are 
effective.

Some suggestions for possible future work:

- The selection methods proposed have been tested considering perfect channel state information available at the transmitter. The performance of the proposed schemes could be evaluated for partial CSI knowledge, obtained by employing channel estimation algorithms.

- Joint transmit and receive selection could be implemented for MIMOOFDM systems.

- Some other non-linear precoding techniques could be employed and tested in MIMO-OFDM systems.

- To evaluate the performance in systems with larger dimensions, using sub-optimum search algorithms, such as the Genetic Algorithm or ITES [11]. 


\section{Bibliography}

[1] LIU, G.; HUANG, Y.; WANG, F.; LIU, J. ; WANG, Q.. 5G features from operation perspective and fundamental performance validation by field trial. China Communications, 15(11):33-50, Nov 2018.

[2] SHAFI, M.; MOLISCH, A. F.; SMITH, P. J.; HAUSTEIN, T.; ZHU, P.; DE SILVA, P.; TUFVESSON, F.; BENJEBBOUR, A. ; WUNDER, G.. 5G: A tutorial overview of standards, trials, challenges, deployment, and practice. IEEE Journal on Selected Areas in Communications, 35(6):12011221, June 2017.

[3] CHOUDHURY, D.. 5G wireless and millimeter wave technology evolution: An overview. In: 2015 IEEE MTT-S INTERNATIONAL MICROWAVE SYMPOSIUM, p. 1-4, May 2015.

[4] ETSI. 5g nr - physical channels and modulation. In: $3^{\text {rd }}$ GENERATION PARTNERSHIP PROJECT (3GPP), Jul 2018.

[5] MUQUET, B.; ZHENGDAO WANG; GIANNAKIS, G. B.; DE COURVILLE, M. ; DUHAMEL, P.. Cyclic prefixing or zero padding for wireless multicarrier transmissions? IEEE Transactions on Communications, 50(12):2136-2148, Dec 2002.

[6] PAUlRAJ, A. J.; GORE, D. A.; NABAR, R. U. ; BOlCSKEI, H.. An overview of MIMO communications - a key to gigabit wireless. Proceedings of the IEEE, 92(2):198-218, Feb 2004.

[7] LARSSON, E. G.; EDFORS, O.; TUFVESSON, F. ; MARZETTA, T. L.. Massive MIMO for next generation wireless systems. IEEE Communications Magazine, 52(2):186-195, February 2014.

[8] LU, L.; LI, G. Y.; SWINDLEHURST, A. L.; ASHIKHMIN, A. ; ZHANG, R.. An overview of massive MIMO: Benefits and challenges. IEEE Journal of Selected Topics in Signal Processing, 8(5):742-758, Oct 2014.

[9] LI, Y. G.; WINTERS, J. H. ; SOlLENBERGER, N. R.. MIMO-OFDM for wireless communications: signal detection with enhanced 
channel estimation. IEEE Transactions on Communications, 50(9):14711477, Sep. 2002.

[10] STUBER, G. L.; BARRY, J. R.; MCLAUGHLIN, S. W.; YE LI; INGRAM, M. A. ; PRATT, T. G.. Broadband MIMO-OFDM wireless communications. Proceedings of the IEEE, 92(2):271-294, Feb 2004.

[11] PÉREZ, D. A.; SAMPAIO-NETO, R.. Bit error rate minimizing antena selection in zero-forcing precoded MU-MIMO systems. In: XXXVI SIMPóSIO BRASILEIRO DE TELECOMUNICAçõES E PROCESSAMENTO DE SINAIS (SBRT 2018), Campina Grande, Brazil, September 2018.

[12] HEATH, R. W.; SANDHU, S. ; PAULRAJ, A.. Antenna selection for spatial multiplexing systems with linear receivers. IEEE Communications Letters, 5(4):142-144, April 2001.

[13] DUARTE, A.; CAL-BRAZ, J. ; SAMPAIO-NETO, R.. Index encoding and antenna selection for GPSM multiuser mimo communication. IEEE Transactions on Wireless Communications, 2019.

[14] CHEN, C.. A computationally efficient near-optimal algorithm for capacity-maximization based joint transmit and receive antenna selection. IEEE Communications Letters, 14(5):402-404, May 2010.

[15] SADEK, M.; TARIGHAT, A. ; SAYED, A. H.. Active antenna selection in multiuser mimo communications. IEEE Transactions on Signal Processing, 55(4):1498-1510, April 2007.

[16] PARK, J.; CHUN, J. ; PARK, H.. Generalised singular value decompositionbased algorithm for multi-user multiple-input multipleoutput linear precoding and antenna selection. Communications, IET, 4:1899-1907, 122010.

[17] KHOROV, E.; KIRYANOV, A.; LYAKHOV, A. ; BIANCHI, G.. A tutorial on IEEE 802.11ax high efficiency WLANs. IEEE Communications Surveys Tutorials, 21(1):197-216, Firstquarter 2019.

[18] GHOSH, A.; MAEDER, A.; BAKER, M. ; CHANDRAMOULI, D.. 5G evolution: A view on 5G cellular technology beyond 3GPP release 15. IEEE Access, 7:127639-127651, 2019.

[19] GESBERT, D.; KOUNTOURIS, M.; HEATH, R. W.; BYOUNG CHAE, C. ; SALZER, T.. Shifting the MIMO paradigm. IEEE Signal Processing Magazine, 24:36-46, October 2007. 
[20] KIM, J.; KANG, C. G.; YANG, W.-Y. ; CHO, Y. S.. MIMO-OFDM Wireless Communications with MATLAB. John Wiley and Sons, 2010.

[21] PATZOLD, M.. MOBILE RADIO CHANNELS. John Wiley and Sons, 2012.

[22] SKLAR, B.. Digital Communications: Fundamentals and Applications 2/E. Prentice Hall, 2002.

[23] RAPPAPORT, T. S.. Wireless Communications: Principles and Practice. Prentice Hall, 2001.

[24] JANKIRAMAN, M.. Space-Time Codes and MIMO Systems. Artech House, 2004.

[25] PAULRAJ, A.; GORE, D. ; NABAR, R.. Introduction to Space-Time Wireless Communications. Cambridge University Press, 2003.

[26] GARCÍA, J. L. A.. Interference Mitigation Schemes for the Uplink of Massive MIMO in 5G Heterogeneous Cellular Networks. Phd thesis, Pontifical Catholic University of Rio, Brazil, March, 2016.

[27] ABBASI, M.; TADAION, A. A. ; TABAN, M. R.. An improvement of ZF/LR method in MIMO detection. In: 6TH INTERNATIONAL SYMPOSIUM ON TELECOMMUNICATIONS (IST), p. 426-430, Nov 2012.

[28] FATEMA, N.; HUA, G.; XIANG, Y.; PENG, D. ; NATGUNANATHAN, I.. Massive MIMO linear precoding: A survey. IEEE Systems Journal, 12(4):3920-3931, Dec 2018.

[29] VU, M.; PAULRAJ, A.. MIMO wireless linear precoding. IEEE SIGNAL PROCESSING MAGAZINE, 86, October 2007.

[30] CLERCKX, B.; OESTGES, C.. MIMO Wireless Networks (Second Edition). Academic Press, 2013.

[31] FLETCHER, R.. Practical Methods of Optimization (Second Edition). John Wiley and Sons, 2000.

[32] STUBER, G. L.; BARRY, J. R.; MCLAUGHLIN, S. W.; YE LI; INGRAM, M. A. ; PRATT, T. G.. Broadband MIMO-OFDM wireless communications. Proceedings of the IEEE, 92(2):271-294, Feb 2004. 
[33] BeRENGUER, I.; WANG, X. ; KRISHNAMURTHY, V.. Adaptive MIMO antenna selection via discrete stochastic optimization. IEEE Transactions on Signal Processing, 53(11), 2005.

[34] CHOI, Y.-S.; MOLISCH, A. F.; WIN, M. Z. ; WINTERS, J. H.. Fast algorithms for antenna selection in MIMO systems. 2003.

[35] GAO, X.; EDFORS, O.; LIU, J. ; TUFVESSON, F.. Antenna selection in measured massive MIMO channels using convex optimization. In: 2013 IEEE GLOBECOM WORKSHOPS (GC WKSHPS), p. 129-134, Dec 2013.

[36] MAHBOOB, S.; RUBY, R. ; LEUNG, V. C. M.. Transmit antenna selection for downlink transmission in a massively distributed antenna system using convex optimization. In: 2012 SEVENTH INTERNATIONAL CONFERENCE ON BROADBAND, WIRELESS COMPUTING, COMMUNICATION AND APPLICATIONS, p. 228-233, Nov 2012.

[37] GORE, D. A.; NABAR, R. U. ; PAULRAJ, A.. Selecting an optimal set of transmit antennas for a low rank matrix channel. In: 2000 IEEE INTERNATIONAL CONFERENCE ON ACOUSTICS, SPEECH, AND SIGNAL PROCESSING. PROCEEDINGS (CAT. NO.00CH37100), volumen 5, p. 2785-2788 vol.5, June 2000 .

[38] SANAYEI, S.; NOSRATINIA, A.. Antenna selection in MIMO systems. IEEE Communications Magazine, 42(10):68-73, Oct 2004.

[39] TAI, T.; CHEN, H.; CHUNG, W. ; LEE, T.. Energy efficient norm-andcorrelation-based antenna selection algorithm in spatially correlated massive multi-user MIMO systems. In: 2017 IEEE INTERNATIONAL WORKSHOP ON SIGNAL PROCESSING SYSTEMS (SIPS), $p$. 1-5, Oct 2017. 


\section{A}

\section{Energy Relations for Multi-User}

The expression in (2-22) can be obtained as follows. From (2-9), we can express the transmission matrix as

$$
T_{P}^{M U}=\sum_{k=1}^{K} \mathcal{D}_{P}^{k} \mathbf{W}_{M}^{*}
$$

and

$$
\sum_{k=1}^{K} \mathcal{D}_{P}^{k}=\left[\begin{array}{llll}
\mathfrak{X}_{1} & \mathfrak{X}_{2} & \ldots & \mathfrak{X}_{M}
\end{array}\right]
$$

The $\mathfrak{X}_{m}$ can be obtained from

$$
\mathfrak{X}_{m}=\sum_{k=1}^{K} \mathfrak{P}_{m}^{k} \mathfrak{s}_{m}^{k}
$$

Thus,

$$
\mathbb{E}\left\{\left\|T_{P}^{M U}\right\|^{2}\right\}=\mathbb{E}\left[\operatorname{Tr}\left\{\left(\sum_{k=1}^{K} \mathcal{D}_{P}^{k}\right) \mathbf{W}_{M}^{*} \mathbf{W}_{M}^{T}\left(\sum_{k=1}^{K} \mathcal{D}_{P}^{k}\right)^{H}\right\}\right]
$$

but $\mathbf{W}_{M}^{*} \mathbf{W}_{M}^{T}=\mathbf{I}_{M}$. Then,

$$
\begin{aligned}
\mathbb{E}\left\{\left\|T_{P}^{M U}\right\|^{2}\right\} & =\mathbb{E}\left[\operatorname{Tr}\left\{\left(\sum_{k=1}^{K} \mathcal{D}_{P}^{k}\right)\left(\sum_{k=1}^{K} \mathcal{D}_{P}^{k}\right)^{H}\right\}\right] \\
& =\mathbb{E}\left[\sum_{m=1}^{M} \mathfrak{X}_{m} \mathfrak{X}_{m}^{H}\right] \\
& =\sum_{m=1}^{M} \mathbb{E}\left\{\left\|\mathfrak{X}_{m}\right\|^{2}\right\} .
\end{aligned}
$$


B

\section{Energy Relations for Single-User}

The average energy available at the transmitter is given by

$$
\begin{aligned}
E_{T} & =\frac{1}{M} \sum_{m=1}^{M} \mathbb{E}\left[\left\|\mathbf{x}_{m}\right\|^{2}\right] \\
& =\frac{1}{M} \sum_{m=1}^{M} \operatorname{Tr}\left\{\mathbb{E}\left[\mathbf{x}_{m} \mathbf{x}_{m}^{H}\right]\right\} \\
& =\frac{1}{M} E_{s} \sum_{m=1}^{M} \operatorname{Tr}\left\{\mathbf{I}_{N_{R F}}\right\} \\
& =E_{s} N_{R F},
\end{aligned}
$$

then the average information symbol energy $E_{s}$ is given by

$$
E_{s}=\frac{E_{T}}{N_{R F}}
$$

